\title{
Structural analysis of synchronization in networks of linear oscillators
}

\author{
S. Emre Tuna*
}

December 30, 2019

\begin{abstract}
In networks of identical linear oscillators (e.g. pendulums undergoing small vibrations) coupled through both dissipative connectors (e.g. dampers) and restorative connectors (e.g. springs) the relation between asymptotic synchronization and coupling structure is studied. Conditions on the interconnection under which synchronization can be achieved for some selection of coupling strengths are established. How to strengthen those conditions so that synchronization is guaranteed for all admissible parameter values is also presented.
\end{abstract}

\section{Introduction}

Consider the coupled array of $q \geq 2$ linear time-invariant (LTI) oscillators

$$
M \ddot{x}_{i}+K x_{i}+B\left(\sum_{j=1}^{q} d_{i j}\left(\dot{y}_{i}-\dot{y}_{j}\right)+\sum_{j=1}^{q} r_{i j}\left(y_{i}-y_{j}\right)\right)=0, \quad y_{i}=B^{T} x_{i}
$$

for $i=1,2, \ldots, q$; where $x_{i} \in \mathbb{R}^{n}, y_{i} \in \mathbb{R}$, the matrices $M, K \in \mathbb{R}^{n \times n}$ are symmetric positive definite, and $B \in \mathbb{R}^{n \times 1}$. Note that the special case $n=1$ describes an assembly of coupled harmonic oscillators 9]. The scalars $d_{i j}=d_{j i} \geq 0$ are the dissipative coupling strengths and $r_{i j}=r_{j i} \geq 0$ the restorative coupling strengths. (We let $d_{i i}=0$ and $r_{i i}=0$.) Therefore the overall coupling throughout the array can be represented by the pair of laplacian matrices

$$
D=\left[\begin{array}{cccc}
\sum_{j} d_{1 j} & -d_{12} & \cdots & -d_{1 q} \\
-d_{21} & \sum_{j} d_{2 j} & \cdots & -d_{2 q} \\
\vdots & \vdots & \ddots & \vdots \\
-d_{q 1} & -d_{q 2} & \cdots & \sum_{j} d_{q j}
\end{array}\right] \text { and } R=\left[\begin{array}{cccc}
\sum_{j} r_{1 j} & -r_{12} & \cdots & -r_{1 q} \\
-r_{21} & \sum_{j} r_{2 j} & \cdots & -r_{2 q} \\
\vdots & \vdots & \ddots & \vdots \\
-r_{q 1} & -r_{q 2} & \cdots & \sum_{j} r_{q j}
\end{array}\right]
$$

A simple illustration of the setup (1) is shown in Fig. 1, where four fourth-order LC oscillators are coupled via an LTI resistor (with conductance $g_{13}$ ) and three LTI inductors (with inductances $\ell_{12}, \ell_{23}, \ell_{34}$ ). The associated laplacian matrices read

$$
D_{1}=\left[\begin{array}{cccc}
g_{13} & 0 & -g_{13} & 0 \\
0 & 0 & 0 & 0 \\
-g_{13} & 0 & g_{13} & 0 \\
0 & 0 & 0 & 0
\end{array}\right] \quad \text { and } \quad R_{1}=\left[\begin{array}{cccc}
\ell_{12}^{-1} & -\ell_{12}^{-1} & 0 & 0 \\
-\ell_{12}^{-1} & \ell_{12}^{-1}+\ell_{23}^{-1} & -\ell_{23}^{-1} & 0 \\
0 & -\ell_{23}^{-1} & \ell_{23}^{-1}+\ell_{34}^{-1} & -\ell_{34}^{-1} \\
0 & 0 & -\ell_{34}^{-1} & \ell_{34}^{-1}
\end{array}\right]
$$

In a recent work [10, under a controllability assumption 1$]$ on the triple $(M, K, B)$, it was shown that

${ }^{*}$ The author is with Department of Electrical and Electronics Engineering, Middle East Technical University, 06800 Ankara, Turkey. Email: etuna@metu.edu.tr

${ }^{1}$ The triple $(M, K, B)$ satisfies: $\operatorname{rank}\left[\begin{array}{c}K-\omega^{2} M \\ B^{T}\end{array}\right]=n$ for all $\omega \in \mathbb{R}_{>0}$. 


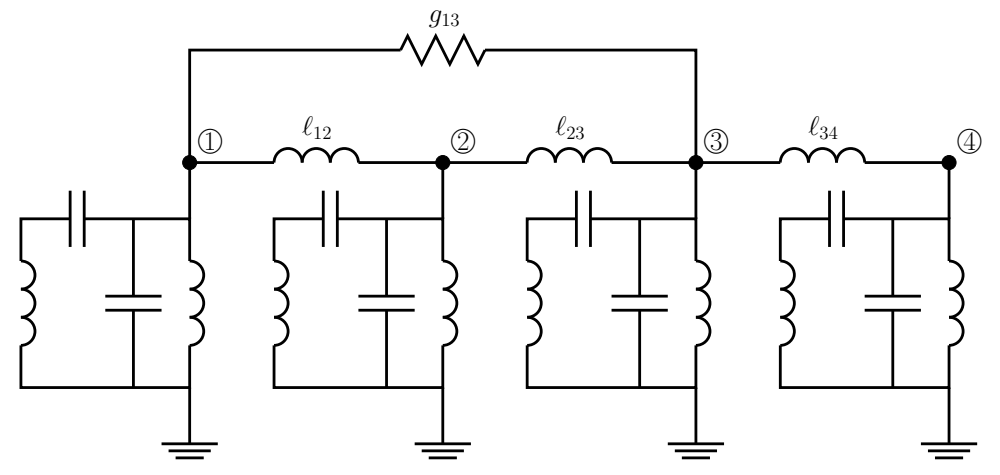

Figure 1: An array of fourth-order oscillators.

Proposition 1 If $\operatorname{Re} \lambda_{2}(D+j R)>d 2$ then (and only then) the oscillators (11) asymptotically synchronize, i.e., $\left\|x_{k}(t)-x_{\ell}(t)\right\| \rightarrow 0$ for all $(k, \ell)$ and all initial conditions.

This means that the pair $(D, R)$ completely characterizes the collective behavior of the array (1) from the synchronization point of view. Be that as it may, one would still like to decipher (to some degree) the condition $\operatorname{Re} \lambda_{2}(D+j R)>0$ in order to expand our understanding on synchronization of oscillators. To this end, in this paper we study the relation between the coupling structure and synchronization. In other words, we investigate conditions on the underlying graphs (disregarding the coupling strengths) that yield synchronization. Therefore our object of inquiry here is the triple $\left(\mathcal{V}, \mathcal{B}_{\mathrm{d}}, \mathcal{B}_{\mathrm{r}}\right)$, where $\mathcal{V}=\left\{\nu_{1}, \nu_{2}, \ldots, \nu_{q}\right\}$ is the set of vertices and the edge sets $\mathcal{B}_{\mathrm{d}}=\left\{\left\{\nu_{i}, \nu_{j}\right\}: d_{i j} \neq 0\right\}$ and $\mathcal{B}_{\mathrm{r}}=\left\{\left\{\nu_{i}, \nu_{j}\right\}: r_{i j} \neq 0\right\}$ represent the dissipative connectors and restorative connectors, respectively. We consider two problems; more properly speaking, two facets of one problem. To describe those facets let us revisit our example circuitry in Fig. 1 under two different scenarios. In the first scenario, suppose that some of the coupling parameters (resistances and inductances) are not exactly known or perhaps subject to change due for instance to the variations of the environment temperature. In such a case Proposition 11 cannot be directly used (because the value of $\operatorname{Re} \lambda_{2}(D+j R)$ is out of reach) and one faces the problem of having to determine synchronization (if possible) by looking at the interconnection $\left(\mathcal{V}, \mathcal{B}_{\mathrm{d}}, \mathcal{B}_{\mathrm{r}}\right)$ only. For certain interconnections it is indeed possible to be sure of asymptotic synchronization without any knowledge (except for their sign) about the coupling strengths. Such interconnections are said to have strong structural synchronization (SSS) property (see Definition 2 for the formal description) and as a part of our analysis here we investigate the conditions guaranteeing such outcome. Another interesting situation is that where the coupling strengths are design parameters (i.e., we are free to choose the resistances and inductances) but the underlying interconnection structure (which oscillator is connected to which and with what type of connector) is predetermined and cannot be altered. When dealing with such a case it is desirable to know beforehand whether the given topology admits a set of parameter values that achieves synchronization. If it does admit then we say that it has structural synchronization (SS) property (see Definition (1) and unearthing the conditions yielding structural synchronization makes the other facet of the problem we study here. Our main findings are listed below.

We establish that an interconnection is $\mathrm{SS3}$ if and only if the union of the two coupling graphs (one of them representing the dissipative coupling, the other representing the restorative coupling) is connected and there exists at least one dissipative connector (Theorem 1). The conditions guaranteeing SSS turn out to be slightly more elaborate. We show that an interconnection is SSS if and only if it admits a sort of flow network, where edge currents and vertex potentials obey certain relatively nontechnical rule:4 (Theorem [3). We also apply this result to some benchmark topologies and obtain simple tests to check SSS property of an interconnection when it is either a path or a cycle or a tree.

The attempts to understand behavioral properties of networks based only on their structures have

\footnotetext{
${ }^{2}$ The definition of $\lambda_{2}(\cdot)$ is given in the next section.

${ }^{3}$ Henceforth we sometimes say an interconnection is SS to mean that it has the SS property.

${ }^{4}$ These rules are listed as (A1), (A2), (A3) later in the paper.
} 
so far focused almost exclusively on the issue of controllability [12, 5. Structural controllability is first addressed in [4. The problem is to determine whether it is possible to obtain a controllable pair $(A, B)$ of matrices (representing an LTI system) under the constraint that certain entries of the matrices must be fixed at zero. Some time later strong structural controllability is introduced in [], where this time the controllability of all admissible pairs is under consideration. Motivated by the emergence of multi-agent systems and the need to control them, the structural controllability theory has recently enjoyed many important developments [8, 14, 11, 3, 7, to name but a few, and found interesting applications, e.g., 13. Despite the rapid advances in network controllability, the relation between coupling structure and synchronization in autonomous networks (i.e., those without control inputs) is relatively an unexplored field. One of the very few works on structural synchronization is [1, where a pair of coupled generalized Lorenz chaotic systems is analyzed theoretically, while the cases with higher number of nodes is studied through numerical experiments. To the best of our knowledge, structural synchronization analysis of coupled LTI oscillators through (generalized) graphs $\left(\mathcal{V}, \mathcal{B}_{\mathrm{d}}, \mathcal{B}_{\mathrm{r}}\right)$ with two different edge sets $\mathcal{B}_{\mathrm{d}}, \mathcal{B}_{\mathrm{r}}$ is absent from the current literature. Our contribution here is therefore intended to be twofold: (i) bringing this void to the attention of researchers and (ii) partially filling it by some preliminary results.

\section{Problem statement}

We introduce some notation first. Let $e_{k} \in \mathbb{R}^{q}$ be the unit vector whose $k$ th entry is 1 , i.e., $e_{k}$ is the $k$ th column of the identity matrix $I$. We let $\mathbf{1}_{q} \in \mathbb{R}^{q}$ denote the vector of all ones. A graph is a pair of sets $(\mathcal{V}, \mathcal{B})$, where $\mathcal{V}=\left\{\nu_{1}, \nu_{2}, \ldots, \nu_{q}\right\}$ is the (nonempty) set of vertices (nodes) and $\mathcal{B}=\left\{\beta_{1}, \beta_{2}, \ldots, \beta_{p}\right\}$ is the set of edges (branches), where each edge is an (unordered) pair $\left\{\nu_{k}, \nu_{\ell}\right\} \subset \mathcal{V}$ of distinct vertices. The graph $(\mathcal{V}, \mathcal{B})$ can be represented by its incidence matrix $G \in \mathbb{R}^{q \times p}$ whose $i$ th column is $g_{i}=e_{k}-e_{\ell}$ (or $g_{i}=e_{\ell}-e_{k}$ ) whenever $\left\{\nu_{k}, \nu_{\ell}\right\}=\beta_{i} \in \mathcal{B}$. Note that when $\mathcal{B}$ is empty, $G$ becomes the $q$-by- 0 empty matrix which (by definition) satisfies: range $G=\{0\} \subset \mathbb{R}^{q}$ and null $G^{T}=\mathbb{R}^{q}$. Two distinct vertices $\nu_{i}, \nu_{j} \in \mathcal{V}$ are said to be connected if $e_{i}-e_{j} \in \operatorname{range} G$. The graph $(\mathcal{V}, \mathcal{B})$ is said to be connected if every pair of distinct vertices is connected, or, equivalently, if null $G^{T}=\operatorname{span}\left\{\mathbf{1}_{q}\right\}$. The connected subgraphs $\left(\mathcal{V}_{1}, \mathcal{B}_{1}\right),\left(\mathcal{V}_{2}, \mathcal{B}_{2}\right), \ldots,\left(\mathcal{V}_{c}, \mathcal{B}_{c}\right)$ are said to be the components of the graph $(\mathcal{V}, \mathcal{B})$ if the edge sets $\mathcal{B}_{i}$ (some of which may be empty) satisfy $\bigcup_{i=1}^{c} \mathcal{B}_{i}=\mathcal{B}$ and the pairwise disjoint vertex sets $\mathcal{V}_{i}$ (some of which may be singleton) satisfy $\bigcup_{i=1}^{c} \mathcal{V}_{i}=\mathcal{V}$. Note that a connected graph has a single component: itself. We also note that the number of components satisfy $c=\operatorname{dim}$ null $G^{T}$. A laplacian matrix $L \in \mathbb{R}^{q \times q}$ associated to the graph $(\mathcal{V}, \mathcal{B})$ has the form $L=G \Lambda G^{T}$, where the $p \times p$ diagonal matrix $\Lambda=\operatorname{diag}\left(w_{1}, w_{2}, \ldots, w_{p}\right)$ stores the edge weights $w_{i}>0$. If $\mathcal{B}=\emptyset$ then we let $L=0$. Note that every laplacian matrix $L$ is symmetric positive semidefinite and that null $L=$ null $G^{T}$. In particular, $L \mathbf{1}_{q}=0$ since $G^{T} \mathbf{1}_{q}=0$. The set of all laplacian matrices associated to the pair $(\mathcal{V}, \mathcal{B})$ is denoted by lap $(\mathcal{V}, \mathcal{B})$. A pair of graphs $\left[\left(\mathcal{V}, \mathcal{B}_{1}\right),\left(\mathcal{V}, \mathcal{B}_{2}\right)\right]$ that share the same vertex set $\mathcal{V}$ defines an interconnection, which we denote by the triple $\left(\mathcal{V}, \mathcal{B}_{1}, \mathcal{B}_{2}\right)$. Given $X \in \mathbb{C}^{q \times q}$, we let $\lambda_{k}(X)$ denote the $k$ th smallest eigenvalue of $X$ with respect to the real part. That is, $\operatorname{Re} \lambda_{1}(X) \leq \operatorname{Re} \lambda_{2}(X) \leq \cdots \leq \operatorname{Re} \lambda_{q}(X) \cdot \operatorname{sgn}(\cdot)$ denotes the sign function.

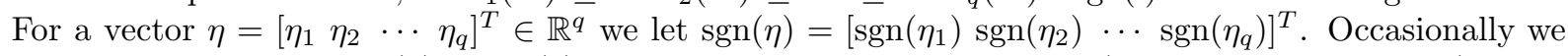
write $\eta \equiv \xi$ in lieu of $\operatorname{sgn}(\eta)=\operatorname{sgn}(\xi)$. The following pair of definitions (based on Proposition 1) is the workhorse of our analysis.

Definition 1 An interconnection $\left(\mathcal{V}, \mathcal{B}_{\mathrm{d}}, \mathcal{B}_{\mathrm{r}}\right)$ is said to have the structural synchronization (SS) property if $\operatorname{Re} \lambda_{2}(D+j R)>0$ for some laplacian matrices $D \in \operatorname{lap}\left(\mathcal{V}, \mathcal{B}_{\mathrm{d}}\right)$ and $R \in \operatorname{lap}\left(\mathcal{V}, \mathcal{B}_{\mathrm{r}}\right)$.

Definition 2 An interconnection $\left(\mathcal{V}, \mathcal{B}_{\mathrm{d}}, \mathcal{B}_{\mathrm{r}}\right)$ is said to have the strong structural synchronization (SSS) property if $\operatorname{Re} \lambda_{2}(D+j R)>0$ for all laplacian matrices $D \in \operatorname{lap}\left(\mathcal{V}, \mathcal{B}_{\mathrm{d}}\right)$ and $R \in \operatorname{lap}\left(\mathcal{V}, \mathcal{B}_{\mathrm{r}}\right)$.

We can now state the problems we study in this paper. Problem 1: Find conditions on the interconnection $\left(\mathcal{V}, \mathcal{B}_{\mathrm{d}}, \mathcal{B}_{\mathrm{r}}\right)$ that guarantee structural synchronization property. Problem 2: Find conditions on the interconnection $\left(\mathcal{V}, \mathcal{B}_{\mathrm{d}}, \mathcal{B}_{\mathrm{r}}\right)$ that guarantee strong structural synchronization property. The solution of the second problem partly depends on that of Problem 1. We therefore study structural synchronization first. 


\section{Structural synchronization}

It turns out to be very easy to check whether a given interconnection is SS or not:

Theorem 1 An interconnection $\left(\mathcal{V}, \mathcal{B}_{\mathrm{d}}, \mathcal{B}_{\mathrm{r}}\right)$ is $S S$ if and only if the graph $\left(\mathcal{V}, \mathcal{B}_{\mathrm{d}} \cup \mathcal{B}_{\mathrm{r}}\right)$ is connected and $\mathcal{B}_{\mathrm{d}}$ is nonempty.

Throughout the rest of this section we will either be proving this result or be busy developing tools (three lemmas) for the proof. Given the simplicity of the statement to be proven, our demonstration seems to be unnecessarily lengthy. It is not unlikely that there is a much shorter (and elegant) proof, but we have so far been unable to discover it.

Lemma 1 Let $(\mathcal{V}, \mathcal{B})$ be a connected graph. Then there exists a laplacian $L \in \operatorname{lap}(\mathcal{V}, \mathcal{B})$ such that no eigenvector of $L$ has a zero entry.

Proof. We prove by induction. Given some connected graph $\Gamma=(\mathcal{V}, \mathcal{B})$, suppose $L \in \operatorname{lap}(\mathcal{V}, \mathcal{B}) \subset \mathbb{R}^{q \times q}$ has no eigenvector with zero entry. This means (being real and symmetric) $L$ has distinct eigenvalues, which we denote by $\sigma_{1}, \sigma_{2}, \ldots, \sigma_{q}$. Moreover, the corresponding unit eigenvectors $u_{1}, u_{2}, \ldots, u_{q} \in \mathbb{R}^{q}$ are pairwise orthogonal. Let us define the positive constants $c_{1}$ and $c_{2}$ as

$$
\begin{aligned}
c_{1} & :=\min _{i \neq j}\left|\sigma_{i}-\sigma_{j}\right|, \\
c_{2} & :=\min _{i, j}\left|e_{i}^{T} u_{j}\right| .
\end{aligned}
$$

Let $\mathcal{V}=\left\{\nu_{1}, \nu_{2}, \ldots, \nu_{q}\right\}$. Now we augment the graph $\Gamma$ by adding a new edge $\left\{\nu_{k}, \nu_{\ell}\right\} \notin \mathcal{B}$. We consider two possibilities that preserve connectedness.

Case 1: $\nu_{k}, \nu_{\ell} \in \mathcal{V}$. In this case the new edge is between two already existing vertices. Let $\mathcal{B}^{+}=$ $\mathcal{B} \cup\left\{\left\{\nu_{k}, \nu_{\ell}\right\}\right\}$. The new graph $\Gamma^{+}=\left(\mathcal{V}, \mathcal{B}^{+}\right)$clearly is connected. Define $b \in \mathbb{R}^{q}$ as $b=e_{k}-e_{\ell}$. Note that $\left\|b b^{T}\right\|=2$, where we work with the (induced) 2-norm. For the new edge choose now some weight $w>0$ satisfying

$$
w<\frac{c_{1} c_{2}}{8 \sqrt{1+c_{2}^{2}}}
$$

and construct the laplacian

$$
L_{1}=L+w b b^{T} .
$$

Note that $L_{1} \in \operatorname{lap}\left(\mathcal{V}, \mathcal{B}^{+}\right)$. Let now $u \in \mathbb{R}^{q}$ be a unit eigenvector of $L_{1}$. By [2, Cor. 8.1.6] we have $L_{1} u=(\sigma+h) u$ for some $\sigma \in\left\{\sigma_{1}, \sigma_{2}, \ldots, \sigma_{q}\right\}$ and $|h| \leq\left\|w b b^{T}\right\|=w\left\|b b^{T}\right\|=2 w$. Without loss of generality we take $\sigma=\sigma_{1}$, i.e., $L_{1} u=\left(\sigma_{1}+h\right) u$. Let $\alpha_{i}=u_{i}^{T} u$. Since $\left\{u_{1}, u_{2}, \ldots, u_{q}\right\}$ is an orthonormal basis for $\mathbb{R}^{q}$ we have $u=\sum_{i=1}^{q} \alpha_{i} u_{i}$. Moreover, $\|u\|=1$ implies $\sum_{i=1}^{q} \alpha_{i}^{2}=1$. We can write

$$
\begin{aligned}
\left\|\sum_{i=2}^{q} \alpha_{i}\left(\sigma_{1}-\sigma_{i}+h\right) u_{i}\right\| & =\left\|-h \alpha_{1} u_{1}+\left(\sigma_{1}+h\right) \sum_{i=1}^{q} \alpha_{i} u_{i}-\sum_{i=1}^{q} \alpha_{i} \sigma_{i} u_{i}\right\| \\
& =\left\|-h \alpha_{1} u_{1}+\left(\sigma_{1}+h\right) \sum_{i=1}^{q} \alpha_{i} u_{i}-L \sum_{i=1}^{q} \alpha_{i} u_{i}\right\| \\
& =\left\|-h \alpha_{1} u_{1}+\left(\sigma_{1}+h\right) u-\left[L_{1}-w b b^{T}\right] u\right\| \\
& =\left\|-h \alpha_{1} u_{1}+L_{1} u-L_{1} u+w b b^{T} u\right\| \\
& \leq|h| \cdot\left|\alpha_{1}\right| \cdot\left\|u_{1}\right\|+w\left\|b b^{T}\right\| \cdot\|u\| \\
& \leq 4 w
\end{aligned}
$$

since $\left|\alpha_{1}\right| \leq 1$ and $\left\|u_{1}\right\|=1$. Now, by (2) we have $w<c_{1} / 4$ yielding $|h| \leq c_{1} / 2$. Therefore

$$
\sum_{i=2}^{q} \alpha_{i}^{2}=\left\|\sum_{i=2}^{q} \alpha_{i} u_{i}\right\|^{2}=\frac{4}{c_{1}^{2}}\left\|\sum_{i=2}^{q} \alpha_{i} \frac{c_{1}}{2} u_{i}\right\|^{2} \leq \frac{4}{c_{1}^{2}}\left\|\sum_{i=2}^{q} \alpha_{i}\left(\sigma_{1}-\sigma_{i}+h\right) u_{i}\right\|^{2} \leq \frac{64 w^{2}}{c_{1}^{2}} .
$$


This allows us to write for all $j \in\{1,2, \ldots, q\}$

$$
\begin{aligned}
\left|e_{j}^{T} u\right| & =\left|e_{j}^{T} \sum_{i=1}^{q} \alpha_{i} u_{i}\right| \geq\left|\alpha_{1} e_{j}^{T} u_{1}\right|-\left|e_{j}^{T} \sum_{i=2}^{q} \alpha_{i} u_{i}\right| \\
& \geq\left|\alpha_{1}\right| \cdot\left|e_{j}^{T} u_{1}\right|-\left\|e_{j}\right\| \cdot \| \sum_{i=2}^{q} \alpha_{i} u_{i}|| \geq c_{2}\left(1-\sum_{i=2}^{q} \alpha_{i}^{2}\right)^{1 / 2}-\left(\sum_{i=2}^{q} \alpha_{i}^{2}\right)^{1 / 2} \\
& \geq c_{2}\left(1-\frac{64 w^{2}}{c_{1}^{2}}\right)^{1 / 2}-\frac{8 w}{c_{1}} .
\end{aligned}
$$

Combining (2) and (3) yields $\left|e_{j}^{T} u\right|>0$ meaning $e_{j}^{T} u \neq 0$, i.e., the eigenvector $u$ has no zero entry.

Case 2: $\nu_{k} \in \mathcal{V}, \nu_{\ell}=\nu_{q+1} \notin \mathcal{V}$. In this case the new edge is between an existing vertex $\nu_{k}$ and a new vertex $\nu_{q+1}$. Let $\mathcal{V}^{+}=\mathcal{V} \cup\left\{\nu_{q+1}\right\}$ and $\mathcal{B}^{+}=\mathcal{B} \cup\left\{\left\{\nu_{k}, \nu_{q+1}\right\}\right\}$. Since $\nu_{k} \in \mathcal{V}$ the new graph $\Gamma^{+}=\left(\mathcal{V}^{+}, \mathcal{B}^{+}\right)$inherits the connectedness of $\Gamma$. Let $b \in \mathbb{R}^{q+1}$ satisfy $b=e_{k}-e_{q+1}$ yielding $\left\|b b^{T}\right\|=2$. For the new edge choose now some weight $w>0$ satisfying

$$
w<\frac{c_{1} c_{2}}{8 \sqrt{1+q+c_{2}^{2}}}
$$

and construct the laplacian

$$
L_{2}=\underbrace{\left[\begin{array}{cc}
L & 0 \\
0 & 0
\end{array}\right]}_{\tilde{L}}+w b b^{T}
$$

Note that $L_{2} \in \operatorname{lap}\left(\mathcal{V}^{+}, \mathcal{B}^{+}\right)$. Since $\tilde{L}$ is block diagonal, its eigenvalues are contained in the union $\left\{\sigma_{1}, \sigma_{2}, \ldots, \sigma_{q}\right\} \cup\{0\}$. Being a laplacian matrix, $L$ already has an eigenvalue at the origin. Therefore $L$ and $\tilde{L}$ share the same eigenvalues. Let now $u \in \mathbb{R}^{q+1}$ be a unit eigenvector of $L_{2}$. We invoke once again [2, Cor. 8.1.6] and write (without loss of generality) $L_{2} u=\left(\sigma_{1}+h\right) u$ for some $|h| \leq 2 w$. Let $\alpha_{1}, \alpha_{2}, \ldots, \alpha_{q+1} \in \mathbb{R}$ be such that

$$
u=\left[\begin{array}{c}
\sum_{i=1}^{q} \alpha_{i} u_{i} \\
\alpha_{q+1}
\end{array}\right]
$$

Since $\|u\|=1$ we have $\sum_{i=1}^{q+1} \alpha_{i}^{2}=1$. Note first that $\alpha_{q+1}$ cannot be zero. To see that suppose otherwise. That is, $u=\left[\begin{array}{ll}\sum_{i=1}^{q} \alpha_{i} u_{i}^{T} & 0\end{array}\right]^{T}$. We could then write

$$
0=\left(\sigma_{1}+h\right) e_{q+1}^{T} u=e_{q+1}^{T}\left(\left[\begin{array}{cc}
L & 0 \\
0 & 0
\end{array}\right]+w b b^{T}\right) u=w\left(e_{q+1}^{T} b\right)\left(b^{T} u\right)
$$

which would imply (since $w>0$ and $e_{q+1}^{T} b=-1$ ) that $b^{T} u=0$ yielding $e_{k}^{T} \sum_{i=1}^{q} \alpha_{i} u_{i}=0$, i.e., the $k$ th entry of the vector $\sum_{i=1}^{q} \alpha_{i} u_{i}$ is zero. But this would be a contradiction because $b^{T} u=0$ implies that $\sum_{i=1}^{q} \alpha_{i} u_{i}$ has to be an eigenvector of $L$ (which has no eigenvector with a zero entry) as can be seen through

$$
\left[\begin{array}{c}
\left(\sigma_{1}+h\right) \sum_{i=1}^{q} \alpha_{i} u_{i} \\
0
\end{array}\right]=\left(\sigma_{1}+h\right) u=\left(\left[\begin{array}{cc}
L & 0 \\
0 & 0
\end{array}\right]+w b b^{T}\right) u=\left[\begin{array}{c}
L \sum_{i=1}^{q} \alpha_{i} u_{i} \\
0
\end{array}\right] .
$$


Having established $\alpha_{q+1} \neq 0$ let us continue by writing

$$
\begin{aligned}
\left\|\left[\begin{array}{c}
\sum_{i=2}^{q} \alpha_{i}\left(\sigma_{1}-\sigma_{i}+h\right) u_{i} \\
\left(\sigma_{1}+h\right) \alpha_{q+1}
\end{array}\right]\right\| & =\left\|-\left[\begin{array}{c}
h \alpha_{1} u_{1} \\
0
\end{array}\right]+\left(\sigma_{1}+h\right)\left[\begin{array}{c}
\sum_{i=1}^{q} \alpha_{i} u_{i} \\
\alpha_{q+1}
\end{array}\right]-\left[\begin{array}{c}
\sum_{i=1}^{q} \alpha_{i} \sigma_{i} u_{i} \\
0
\end{array}\right]\right\| \\
& =\left\|-\left[\begin{array}{c}
h \alpha_{1} u_{1} \\
0
\end{array}\right]+\left(\sigma_{1}+h\right) u-\tilde{L} u\right\| \\
& =\left\|-\left[\begin{array}{c}
h \alpha_{1} u_{1} \\
0
\end{array}\right]+L_{2} u-\left[L_{2}-w b b^{T}\right] u\right\| \\
& =\left\|-\left[\begin{array}{c}
h \alpha_{1} u_{1} \\
0
\end{array}\right]+w b b^{T} u\right\| \\
& \leq|h| \cdot\left|\alpha_{1}\right| \cdot\left\|u_{1}\right\|+w\left\|b b^{T}\right\| \cdot\|u\| \\
& \leq 4 w .
\end{aligned}
$$

We now study two subcases. Subcase 2.1: $\sigma_{1}>0$. Note that since $0 \in\left\{\sigma_{1}, \sigma_{2}, \ldots, \sigma_{q}\right\}$ we have $\sigma_{1} \geq c_{1}$. By (5D) we can therefore write

$$
\sum_{i=2}^{q+1} \alpha_{i}^{2}=\left\|\left[\begin{array}{c}
\sum_{i=2}^{q} \alpha_{i} u_{i} \\
\alpha_{q+1}
\end{array}\right]\right\|^{2}=\frac{4}{c_{1}^{2}}\left\|\left[\begin{array}{c}
\sum_{i=2}^{q} \frac{c_{1}}{2} \alpha_{i} u_{i} \\
\frac{c_{1}}{2} \alpha_{q+1}
\end{array}\right]\right\|^{2} \leq \frac{4}{c_{1}^{2}}\left\|\left[\begin{array}{c}
\sum_{i=2}^{q} \alpha_{i}\left(\sigma_{1}-\sigma_{i}+h\right) u_{i} \\
\left(\sigma_{1}+h\right) \alpha_{q+1}
\end{array}\right]\right\|^{2} \leq \frac{64 w^{2}}{c_{1}^{2}} .
$$

This lets us have for all $j \in\{1,2, \ldots, q\}$

$$
\begin{aligned}
\left|e_{j}^{T} u\right| & =\left|e_{j}^{T} \sum_{i=1}^{q} \alpha_{i} u_{i}\right| \geq\left|\alpha_{1} e_{j}^{T} u_{1}\right|-\left|e_{j}^{T} \sum_{i=2}^{q} \alpha_{i} u_{i}\right| \\
& \geq\left|\alpha_{1}\right| \cdot\left|e_{j}^{T} u_{1}\right|-\left\|e_{j}\right\| \cdot \| \sum_{i=2}^{q} \alpha_{i} u_{i}|| \geq c_{2}\left(1-\sum_{i=2}^{q+1} \alpha_{i}^{2}\right)^{1 / 2}-\left(\sum_{i=2}^{q} \alpha_{i}^{2}\right)^{1 / 2} \\
& \geq c_{2}\left(1-\frac{64 w^{2}}{c_{1}^{2}}\right)^{1 / 2}-\frac{8 w}{c_{1}} .
\end{aligned}
$$

Combining (44) and (6) yields $\left|e_{j}^{T} u\right|>0$ meaning $e_{j}^{T} u \neq 0$, i.e., the first $q$ entries of the eigenvector $u$ are nonzero. Recall that the last entry $\alpha_{q+1}$ is nonzero, too. Therefore $u$ has no zero entry. Subcase 2.2: $\sigma_{1}=0$. When $\sigma_{1}=0$ we have $L_{2} u=h u$. That $L_{2}$ is symmetric positive semidefinite implies that the eigenvalue $h$ is nonnegative. It turns out that the two possibilities $(h>0$ or $h=0)$ require slightly different treatments. We begin by $h>0$. Recall that $L_{2} \mathbf{1}_{q+1}=0$. Since $h \neq 0$ and $L_{2}$ is symmetric, the eigenvectors $u$ and $\mathbf{1}_{q+1}$ have to be orthogonal. Moreover, $\sigma_{1}=0$ yields $u_{1}=\mathbf{1}_{q} / \sqrt{q}$ since we know that $L \mathbf{1}_{q}=0$ and the eigenvalue of $L$ at the origin cannot be repeated. Thence we can write $\mathbf{1}_{q}^{T} u_{i}=0$ for $i=2,3, \ldots, q$. This implies $\alpha_{q+1}^{2}=q \alpha_{1}^{2}$ because

$$
\sqrt{q} \alpha_{1}+\alpha_{q+1}=\left(\mathbf{1}_{q}^{T} u_{1}\right) \alpha_{1}+\alpha_{q+1}+\sum_{i=2}^{q} \alpha_{i}\left(\mathbf{1}_{q}^{T} u_{i}\right)=\alpha_{q+1}+\mathbf{1}_{q}^{T} \sum_{i=1}^{q} \alpha_{i} u_{i}=\mathbf{1}_{q+1}^{T} u=0 .
$$

Therefore

$$
\alpha_{1}^{2}=\frac{\alpha_{1}^{2}+\alpha_{q+1}^{2}}{q+1}=\frac{1-\sum_{i=2}^{q} \alpha_{i}^{2}}{q+1}
$$

Now, by (5) we can write

$$
\sum_{i=2}^{q} \alpha_{i}^{2}=\left\|\sum_{i=2}^{q} \alpha_{i} u_{i}\right\|^{2}=\frac{4}{c_{1}^{2}}\left\|\sum_{i=2}^{q} \frac{c_{1}}{2} \alpha_{i} u_{i}\right\|^{2} \leq \frac{4}{c_{1}^{2}}\left\|\left[\begin{array}{c}
\sum_{i=2}^{q} \alpha_{i}\left(\sigma_{1}-\sigma_{i}+h\right) u_{i} \\
\left(\sigma_{1}+h\right) \alpha_{q+1}
\end{array}\right]\right\|^{2} \leq \frac{64 w^{2}}{c_{1}^{2}} .
$$


This and (7) allow us write for all $j \in\{1,2, \ldots, q\}$

$$
\begin{aligned}
\left|e_{j}^{T} u\right| & =\left|e_{j}^{T} \sum_{i=1}^{q} \alpha_{i} u_{i}\right| \geq\left|\alpha_{1} e_{j}^{T} u_{1}\right|-\left|e_{j}^{T} \sum_{i=2}^{q} \alpha_{i} u_{i}\right| \\
& \geq\left|\alpha_{1}\right| \cdot\left|e_{j}^{T} u_{1}\right|-\left\|e_{j}\right\| \cdot \| \sum_{i=2}^{q} \alpha_{i} u_{i}|| \geq \frac{c_{2}}{\sqrt{q+1}}\left(1-\sum_{i=2}^{q} \alpha_{i}^{2}\right)^{1 / 2}-\left(\sum_{i=2}^{q} \alpha_{i}^{2}\right)^{1 / 2} \\
& \geq \frac{c_{2}}{\sqrt{q+1}}\left(1-\frac{64 w^{2}}{c_{1}^{2}}\right)^{1 / 2}-\frac{8 w}{c_{1}} .
\end{aligned}
$$

Combining (44) and (8) yields $\left|e_{j}^{T} u\right|>0$ whence follows that the first $q$ entries of $u$ are nonzero. Since we also have $\alpha_{q+1} \neq 0$, the eigenvector $u$ is free of any zero entry. Finally, we deal with the other case $h=0$. That is, $L_{2} u=0$. Since the laplacian $L_{2}$ represents a connected graph, dim null $L_{2}=1$, whence the eigenvector corresponding to the eigenvalue at the origin is unique (up to a scaling). More precisely, $u=\mathbf{1}_{q+1} / \sqrt{q+1}$, meaning $u$ has no zero entry.

To recapitulate, we have established the following. If for a given connected graph $\Gamma=(\mathcal{V}, \mathcal{B})$ there exists some laplacian $L \in \operatorname{lap}(\mathcal{V}, \mathcal{B})$ such that no eigenvector of $L$ has a zero entry, then for any connected graph $\Gamma^{+}$that can be constructed from $\Gamma$ by adding a new edge, there must also exist a laplacian with the same property. Since (i) any connected graph can be gradually constructed in this edge-by-edge fashion starting from a single edge connected graph $\Gamma_{0}=\left(\left\{\nu_{1}, \nu_{2}\right\},\left\{\left\{\nu_{1}, \nu_{2}\right\}\right\}\right)$ and (ii) the eigenvectors of any laplacian $L_{0} \in \operatorname{lap} \Gamma_{0}$ are

$$
u_{1}=\left[\begin{array}{c}
\frac{1}{\sqrt{2}} \\
\frac{1}{\sqrt{2}}
\end{array}\right] \quad \text { and } \quad u_{2}=\left[\begin{array}{r}
\frac{1}{\sqrt{2}} \\
-\frac{1}{\sqrt{2}}
\end{array}\right]
$$

(i.e., they have no zero entries) the result follows by induction.

Lemma 2 Let $D, R \in \mathbb{R}^{q \times q}$ be symmetric positive semidefinite matrices. Then the complex matrix $D+j R$ has no eigenvalue on the open left half plane.

Proof. Let $\lambda \in \mathbb{C}$ be an eigenvalue of $D+j R$ with the corresponding unit eigenvector $u \in \mathbb{C}^{q}$. We can write $\operatorname{Re} \lambda=\operatorname{Re}\left(u^{*} \lambda u\right)=\operatorname{Re}\left(u^{*}[D+j R] u\right)=\operatorname{Re}\left(u^{*} D u+j u^{*} R u\right)=u^{*} D u \geq 0$. Hence the result.

Lemma 3 Let $D, R \in \mathbb{R}^{q \times q}$ be symmetric positive semidefinite matrices satisfying $D \mathbf{1}_{q}=R \mathbf{1}_{q}=0$. Then $\operatorname{Re} \lambda_{2}(D+j R)>0$ if and only if $R$ has no eigenvector in the set null $D \backslash \operatorname{span}\left\{\mathbf{1}_{q}\right\}$.

Proof. Suppose $\operatorname{Re} \lambda_{2}(D+j R) \leq 0$. By Lemma 2 this implies that $D+j R$ has at least two eigenvalues on the imaginary axis. We already know that one of these eigenvalues is at the origin since $[D+$ $j R] \mathbf{1}_{q}=0$. Let $\lambda=j \sigma$ with $\sigma \in \mathbb{R}$ be the other eigenvalue. We claim that there exists an eigenvector $u \notin \operatorname{span}\left\{\mathbf{1}_{q}\right\}$ satisfying $[D+j R] u=j \sigma u$. This is obvious when $\sigma \neq 0$. It is still true when $\sigma=0$ (i.e., when the eigenvalue at the origin is repeated) because otherwise $\mathbf{1}_{q}$ would be the only eigenvector for the eigenvalue at the origin, which would imply the existence of a generalized eigenvector $v$ satisfying $[D+j R] v=\mathbf{1}_{q}$. But, since $D$ and $R$ are symmetric, this would result in the following contradiction: $q=\mathbf{1}_{q}^{T} \mathbf{1}_{q}=\mathbf{1}_{q}^{T}[D+j R] v=\left([D+j R] \mathbf{1}_{q}\right)^{T} v=0$. Now, without loss of generality let $\|u\|=1$. We can write $j \sigma=u^{*}(j \sigma u)=u^{*}[D+j R] u=u^{*} D u+j u^{*} R u$ which implies $u^{*} D u=0$ and then $D u=0$ due to the fact that $D$ and $R$ are symmetric positive semidefinite. That $u$ belongs to null $D$ means that $R u=\sigma u$, i.e., it is an eigenvector of $R$, since $j \sigma u=[D+j R] u=j R u$. Hence we have established that if the condition $\operatorname{Re} \lambda_{2}(D+j R)>0$ fails to hold then $R$ must have an eigenvector in the set null $D \backslash \operatorname{span}\left\{\mathbf{1}_{q}\right\}$.

Now we show the other direction. Suppose $R$ has an eigenvector $z \notin \operatorname{span}\left\{\mathbf{1}_{q}\right\}$ satisfying $D z=0$. Let $\lambda$ be the corresponding eigenvalue, which is real since $R$ is symmetric positive semidefinite. This implies $j \lambda$ is an eigenvalue of $D+j R$ since $[D+j R] z=D z+j R z=j \lambda z$. Then combining $z \notin \operatorname{span}\left\{\mathbf{1}_{q}\right\}$ and $[D+j R] \mathbf{1}_{q}=0$ allows us to see that $D+j R$ has at least two eigenvalues on the imaginary axis. This at once yields $\operatorname{Re} \lambda_{2}(D+j R) \leq 0$.

Proof of Theorem 1. We prove necessity first. Let $\left(\mathcal{V}, \mathcal{B}_{\mathrm{d}}, \mathcal{B}_{\mathrm{r}}\right)$ be an SS interconnection. This means that we can find laplacians $D \in \operatorname{lap}\left(\mathcal{V}, \mathcal{B}_{\mathrm{d}}\right)$ and $R \in \operatorname{lap}\left(\mathcal{V}, \mathcal{B}_{\mathrm{r}}\right)$ satisfying $\operatorname{Re} \lambda_{2}(D+j R)>0$. Now, 
we claim that $\mathcal{B}_{\mathrm{d}}$ cannot be empty, for otherwise $D=0$ and $\operatorname{Re} \lambda_{2}(D+j R)=\operatorname{Re} \lambda_{2}(j R)=0$ since all the eigenvalues of $j R$ are purely imaginary thanks to that all the eigenvalues of $R \in \mathbb{R}^{q \times q}$ are real due to symmetry $R=R^{T}$. Furthermore, the graph $\Gamma=\left(\mathcal{V}, \mathcal{B}_{\mathrm{d}} \cup \mathcal{B}_{\mathrm{r}}\right)$ must be connected. To see that suppose $\Gamma$ is not connected. Let $G$ be the incidence matrix representing $\Gamma$. By construction we have $\mathbf{1}_{q} \in \operatorname{null} G^{T}$, however null $G^{T} \neq \operatorname{span}\left\{\mathbf{1}_{q}\right\}$ because $\Gamma$ is not connected. Hence the null space of $G^{T}$ must be of dimension two or larger. This allows us to be able to find a vector $v \in \operatorname{null} G^{T} \backslash \operatorname{span}\left\{\mathbf{1}_{q}\right\}$. Let $G_{\mathrm{d}}$ and $G_{\mathrm{r}}$ be the incidence matrices of the graphs $\left(\mathcal{V}, \mathcal{B}_{\mathrm{d}}\right)$ and $\left(\mathcal{V}, \mathcal{B}_{\mathrm{r}}\right)$, respectively. Note that by construction null $G_{\mathrm{d}}^{T}=\operatorname{null} D$ and null $G_{\mathrm{r}}^{T}=\operatorname{null} R$. Since $G$ is the incidence matrix of $\Gamma=\left(\mathcal{V}, \mathcal{B}_{\mathrm{d}} \cup \mathcal{B}_{\mathrm{r}}\right)$ we can write null $G_{\mathrm{d}}^{T} \cap \operatorname{null} G_{\mathrm{r}}^{T}=\operatorname{null} G^{T} \supset\left\{\mathbf{1}_{q}, v\right\}$. Therefore $(D+j R) \mathbf{1}_{q}=(D+j R) v=0$. This implies $D+j R$ has at least two eigenvalues at the origin thanks to the linear independence of $v$ and $\mathbf{1}_{q}$. We therefore have to have $\operatorname{Re} \lambda_{2}(D+j R) \leq 0$, which contradicts our starting point $\operatorname{Re} \lambda_{2}(D+j R)>0$.

We establish sufficiency through construction. Let the graph $\left(\mathcal{V}, \mathcal{B}_{\mathrm{d}} \cup \mathcal{B}_{\mathrm{r}}\right)$ be connected and $\mathcal{B}_{\mathrm{d}}$ be nonempty. Consider the graph $\Gamma_{\mathrm{r}}=\left(\mathcal{V}, \mathcal{B}_{\mathrm{r}}\right)$. We study two cases. Case 1: $\Gamma_{\mathrm{r}}$ is not connected. Let $c \leq q$ be the number of (connected) components of $\Gamma_{\mathrm{r}}$. (Since $\Gamma_{\mathrm{r}}$ is not connected we have $c \geq 2$.) Let the pairs $\left(\mathcal{V}_{1}, \mathcal{B}_{1}\right),\left(\mathcal{V}_{2}, \mathcal{B}_{2}\right), \ldots,\left(\mathcal{V}_{c}, \mathcal{B}_{c}\right)$ denote these components. For each $i=1,2, \ldots, c$ choose now some laplacian $R_{i} \in \operatorname{lap}\left(\mathcal{V}_{i}, \mathcal{B}_{i}\right) \subset \mathbb{R}^{q_{i} \times q_{i}}$ that has no eigenvector with zero entry. Such choice exists thanks to Lemma 1 Furthermore, let the following condition hold on the collection $\left\{R_{1}, R_{2}, \ldots, R_{c}\right\}$ : if $\lambda$ is a common eigenvalue of $R_{i}$ and $R_{j}(i \neq j)$ then $\lambda=0$. We can impose such a condition (without violating the condition on the eigenevectors) since $R_{i} \in \operatorname{lap}\left(\mathcal{V}_{i}, \mathcal{B}_{i}\right)$ implies $\alpha R_{i} \in \operatorname{lap}\left(\mathcal{V}_{i}, \mathcal{B}_{i}\right)$ for any positive scalar $\alpha$, which we may use to relocate the nonzero eigenvalues of $R_{i}$ without disturbing its eigenvectors. Let us now construct the block diagonal matrix $R=\operatorname{blkdiag}\left(R_{1}, R_{2}, \ldots, R_{c}\right)$. Note that $R \in \operatorname{lap}\left(\mathcal{V}, \mathcal{B}_{\mathrm{r}}\right)$. Let now $z \notin \operatorname{span}\left\{\mathbf{1}_{q}\right\}$ be an eigenvector of $R$ with the corresponding eigenvalue $\lambda$, i.e., $R z=\lambda z$. Since $R$ is positive semidefinite, $\lambda \geq 0$. Let $G_{\mathrm{d}}$ be the incidence matrix of the graph $\left(\mathcal{V}, \mathcal{B}_{\mathrm{d}}\right)$. Choose $\Lambda_{\mathrm{d}}=I$ and construct the laplacian $D=G_{\mathrm{d}} G_{\mathrm{d}}^{T}=G_{\mathrm{d}} \Lambda_{\mathrm{d}} G_{\mathrm{d}}^{T} \in \operatorname{lap}\left(\mathcal{V}, \mathcal{B}_{\mathrm{d}}\right)$. We claim that $D z \neq 0$, which is equivalent to $G_{\mathrm{d}}^{T} z \neq 0$. To prove our claim we study two subcases. Subcase 1.1: $\lambda>0$. Since the set of eigenvalues of the block diagonal matrix $R$ is the union of sets of eigenvalues of its individual blocks and no two blocks share a nonzero eigenvalue, $\lambda$ must be an eigenvalue of one (and only one) of the blocks $R_{1}, R_{2}, \ldots, R_{c}$. Without loss of generality let that block be $R_{1} \in \mathbb{R}^{q_{1} \times q_{1}}$. Hence the eigenvector $z$ must be of the form $z=\left[\begin{array}{ll}z_{1}^{T} & 0\end{array}\right]^{T}$ where $z_{1} \in \mathbb{R}^{q_{1}}$ satisfies $R_{1} z_{1}=\lambda z_{1}$, i.e., $z_{1}$ is an eigenvector of $R_{1}$. Now, since $\left(\mathcal{V}, \mathcal{B}_{\mathrm{d}} \cup \mathcal{B}_{\mathrm{r}}\right)$ is connected there has to be an edge $\left\{\nu_{i}, \nu_{j}\right\} \in \mathcal{B}_{\mathrm{d}}$ that extends between the first component $\left(\mathcal{V}_{1}, \mathcal{B}_{1}\right)$ and another one, i.e., the indices $i, j$ should satisfy $i \in\left\{1,2, \ldots, q_{1}\right\}$ and $j \notin\left\{1,2, \ldots, q_{1}\right\}$. This implies that the incidence matrix $G_{\mathrm{d}}$ must have a column of the form $b=e_{i}-e_{j}$ (or $b=e_{j}-e_{i}$ ) with $i \in\left\{1,2, \ldots, q_{1}\right\}$ and $j \notin\left\{1,2, \ldots, q_{1}\right\}$. Hence $b^{T} z=\left(e_{i}-e_{j}\right)^{T}\left[\begin{array}{ll}z_{1}^{T} & 0\end{array}\right]^{T}=e_{i}^{T} z_{1} \neq 0$ since $z_{1}$ has no zero entry. This yields $G_{\mathrm{d}}^{T} z \neq 0$, which in turn yields $D z \neq 0$. Subcase 1.2: $\lambda=0$. In this case $z$ satisfies $R z=0$ meaning $z \in \operatorname{null} G_{\mathrm{r}}^{T}$, where $G_{\mathrm{r}}$ is the incidence matrix of the graph $\left(\mathcal{V}, \mathcal{B}_{\mathrm{r}}\right)$. To show $D z \neq 0$ suppose otherwise, i.e., $D z=0$, which implies $z \in \operatorname{null} G_{\mathrm{d}}^{T}$. Therefore $z \in \operatorname{null} G_{\mathrm{d}}^{T} \cap \operatorname{null} G_{\mathrm{r}}^{T}=\operatorname{null} G^{T}$ where $G$ is the incidence matrix of the graph $\left(\mathcal{V}, \mathcal{B}_{\mathrm{d}} \cup \mathcal{B}_{\mathrm{r}}\right)$. Since $\left(\mathcal{V}, \mathcal{B}_{\mathrm{d}} \cup \mathcal{B}_{\mathrm{r}}\right)$ is connected, we have null $G^{T}=\operatorname{span}\left\{\mathbf{1}_{q}\right\}$ but this implies $z \in \operatorname{span}\left\{\mathbf{1}_{q}\right\}$, contradicting our earlier assumption that $z \notin \operatorname{span}\left\{\mathbf{1}_{q}\right\}$. This completes the proof of our claim $D z \neq 0$. Hence we have established the following: $R$ can have no eigenvector $z$ in the set null $D \backslash \operatorname{span}\left\{\mathbf{1}_{q}\right\}$. Lemma 3 then yields that the network $\left(\mathcal{V}, \mathcal{B}_{\mathrm{d}}, \mathcal{B}_{\mathrm{r}}\right)$ is SS.

Case 2: $\Gamma_{\mathrm{r}}$ is connected. Note that if $q=2$ then null $D=\operatorname{span}\left\{\mathbf{1}_{q}\right\}$ for all $D \in \operatorname{lap}\left(\mathcal{V}, \mathcal{B}_{\mathrm{d}}\right)$, yielding null $D \backslash \operatorname{span}\left\{\mathbf{1}_{q}\right\}=\emptyset$. The result then trivially follows by Lemma 3 , Thus we henceforth let $q \geq 3$. Choose some edge from the set $\mathcal{B}_{\mathrm{d}}$. Without loss of generality let this edge be labeled $\left\{\nu_{q_{1}}, \nu_{q_{1}+1}\right\}$, where $q_{1} \in\{1,2, \ldots, q-1\}$. Then choose some disjoint vertex sets $\mathcal{V}_{1}, \mathcal{V}_{2} \in \mathcal{V}$ and edge sets $\mathcal{B}_{1}, \mathcal{B}_{2} \in \mathcal{B}$ such that the pairs $\left(\mathcal{V}_{1}, \mathcal{B}_{1}\right)$ and $\left(\mathcal{V}_{2}, \mathcal{B}_{2}\right)$ are connected subgraphs, $\nu_{q_{1}} \in \mathcal{V}_{1}, \nu_{q_{1}+1} \in \mathcal{V}_{2}$, and $\mathcal{V}_{1} \cup \mathcal{V}_{2}=\mathcal{V}$. Obtain now the edge set $\mathcal{B}_{\mathrm{r}}^{-}:=\mathcal{B}_{\mathrm{r}} \backslash\left(\mathcal{B}_{1} \cup \mathcal{B}_{2}\right)$. Since $\left(\mathcal{V}, \mathcal{B}_{\mathrm{r}}\right)$ is connected the new edge set $\mathcal{B}_{\mathrm{r}}^{-}$is a strict subset of $\mathcal{B}_{\mathrm{r}}$. Moreover, the graph $\left(\mathcal{V}, \mathcal{B}_{\mathrm{r}}^{-}\right)$has exactly two (connected) components: $\left(\mathcal{V}_{1}, \mathcal{B}_{1}\right)$ and $\left(\mathcal{V}_{2}, \mathcal{B}_{2}\right)$, where without loss of generality we can let $\mathcal{V}_{1}=\left\{\nu_{1}, \nu_{2}, \ldots, \nu_{q_{1}}\right\}$ and $\mathcal{V}_{2}=$ $\left\{\nu_{q_{1}+1}, \nu_{q_{1}+2}, \ldots, \nu_{q}\right\}$. Let now $q_{2}:=q-q_{1}$ and choose some $R_{1} \in \operatorname{lap}\left(\mathcal{V}_{1}, \mathcal{B}_{1}\right)$ and $R_{2} \in \operatorname{lap}\left(\mathcal{V}_{2}, \mathcal{B}_{2}\right)$ that have no eigenvectors with zero entry. Such $R_{1} \in \mathbb{R}^{q_{1} \times q_{1}}$ and $R_{2} \in \mathbb{R}^{q_{2} \times q_{2}}$ exist thanks to Lemma 1 , Construct the $q \times q$ laplacians $R^{-}=\operatorname{blkdiag}\left(R_{1}, R_{2}\right) \in \operatorname{lap}\left(\mathcal{V}, \mathcal{B}_{\mathrm{r}}^{-}\right)$and $D=G_{\mathrm{d}} G_{\mathrm{d}}^{T} \in \operatorname{lap}\left(\mathcal{V}, \mathcal{B}_{\mathrm{d}}\right)$, where $G_{\mathrm{d}}$ is the incidence matrix of $\left(\mathcal{V}, \mathcal{B}_{\mathrm{d}}\right)$. By earlier analysis we know that $R^{-}$can have no eigenvector in the set null $D \backslash \operatorname{span}\left\{\mathbf{1}_{q}\right\}$. Let $\sigma_{1}, \sigma_{2}, \ldots, \sigma_{q_{1}}$ be the eigenvalues of $R_{1}$ with the corresponding unit eigenvectors $u_{1}, u_{2}, \ldots, u_{q-1} \in \mathbb{R}^{q_{1}}$. Since $R_{1}$ has no eigenvector with zero entry, these eigenvalues are 
distinct. Consequently, the eigenvectors are pairwise orthogonal since $R_{1}$ is symmetric. Likewise, we let $\sigma_{q_{1}+1}, \sigma_{q_{1}+2}, \ldots, \sigma_{q}$ be the distinct eigenvalues of $R_{2}$ with the corresponding pairwise orthogonal unit eigenvectors $u_{q_{1}+1}, u_{q_{1}+2}, \ldots, u_{q} \in \mathbb{R}^{q_{2}}$. Also, without loss of generality (see the explanation above) we assume $\sigma_{i} \neq \sigma_{j}$ (when $\left.i \neq j\right)$ unless $\sigma_{i}=\sigma_{j}=0$. Note that the set of eigenvalues of $R^{-}$is the union of sets of eigenvalues of $R_{1}$ and $R_{2}$. Define the positive constants $c_{1}, c_{2}$ as

$$
\begin{aligned}
c_{1} & :=\min _{\sigma_{i} \neq \sigma_{j}}\left|\sigma_{i}-\sigma_{j}\right|, \\
c_{2} & :=\min _{z \in \mathcal{C}}\|D z\|
\end{aligned}
$$

where we let $\mathcal{C}=\left\{z \in \mathbb{R}^{q}:\|z\|=1, R^{-} z=\sigma_{i} z\right.$ for some $i$, and $\left.\mathbf{1}_{q}^{T} z=0\right\}$. Let $B$ be the incidence matrix of the graph $\left(\mathcal{V}, \mathcal{B}_{\mathrm{r}} \backslash \mathcal{B}_{\mathrm{r}}^{-}\right)$. Choose some $w>0$ satisfying

$$
w<\frac{c_{1} c_{2}}{4\|B\|^{2} \sqrt{c_{2}^{2}+\|D\|^{2}}}
$$

and construct the laplacian

$$
R=R^{-}+w B B^{T}
$$

Note that $R \in \operatorname{lap}\left(\mathcal{V}, \mathcal{B}_{\mathrm{r}}\right)$. Therefore, since $\left(\mathcal{V}, \mathcal{B}_{\mathrm{r}}\right)$ is connected, the eigenvalue of $R$ at the origin is not repeated. Let now $u \in \mathbb{R}^{q}$ be a unit eigenvector of $R$ satisfying $u \notin \operatorname{span}\left\{\mathbf{1}_{q}\right\}$. Being a laplacian, $R$ satisfies $R \mathbf{1}_{q}=0$, i.e., $\mathbf{1}_{q}$ is the eigenvector for the eigenvalue at the origin. Hence $\mathbf{1}_{q}^{T} u=0$ because $R \in \mathbb{R}^{q \times q}$ is symmetric and the eigenvectors $\mathbf{1}_{q}$ and $u$ correspond to different eigenvalues. By [2, Cor. 8.1.6] we have $R u=(\sigma+h) u$ for some $\sigma \in\left\{\sigma_{1}, \sigma_{2}, \ldots, \sigma_{q}\right\}$ and $|h| \leq\left\|w B B^{T}\right\|=w\|B\|^{2}$. Without loss of generality we take $\sigma=\sigma_{1}$, i.e., $R u=\left(\sigma_{1}+h\right) u$. Let $\alpha_{1}, \alpha_{2}, \ldots, \alpha_{q} \in \mathbb{R}$ be such that

$$
u=\left[\begin{array}{c}
\sum_{i=1}^{q_{1}} \alpha_{i} u_{i} \\
\sum_{i=q_{1}+1}^{q} \alpha_{i} u_{i}
\end{array}\right] .
$$

Since $\|u\|=1$ we have $\sum_{i=1}^{q} \alpha_{i}^{2}=1$. We can write

$$
\begin{aligned}
\left\|\left[\begin{array}{c}
\sum_{i=2}^{q_{1}} \alpha_{i}\left(\sigma_{1}-\sigma_{i}+h\right) u_{i} \\
\sum_{i=q_{1}+1}^{q} \alpha_{i}\left(\sigma_{1}-\sigma_{i}+h\right) u_{i}
\end{array}\right]\right\| & =\left\|-\left[\begin{array}{c}
h \alpha_{1} u_{1} \\
0
\end{array}\right]+\left(\sigma_{1}+h\right)\left[\begin{array}{c}
\sum_{i=1}^{q_{1}} \alpha_{i} u_{i} \\
\sum_{i=q_{1}+1}^{q} \alpha_{i} u_{i}
\end{array}\right]-\left[\begin{array}{c}
\sum_{i=1}^{q_{1}} \alpha_{i} \sigma_{i} u_{i} \\
\sum_{i=q_{1}+1}^{q} \alpha_{i} \sigma_{i} u_{i}
\end{array}\right]\right\| \\
& =\left\|-\left[\begin{array}{c}
h \alpha_{1} u_{1} \\
0
\end{array}\right]+\left(\sigma_{1}+h\right) u-R^{-} u\right\| \\
& =\left\|-\left[\begin{array}{c}
h \alpha_{1} u_{1} \\
0
\end{array}\right]+R u-\left[R-w B B^{T}\right] u\right\| \\
& =\left\|-\left[\begin{array}{c}
h \alpha_{1} u_{1} \\
0
\end{array}\right]+w B B^{T} u\right\| \\
& \leq|h| \cdot\left|\alpha_{1}\right| \cdot\left\|u_{1}\right\|+w\left\|B B^{T}\right\| \cdot\|u\| \\
& \leq 2 w\|B\|^{2} .
\end{aligned}
$$

We now study two subcases. Subcase 2.1: $\sigma_{1}>0$. Note that by (9) we have $w<c_{1} /\left(4\|B\|^{2}\right)$ from which follows $|h| \leq c_{1} / 2$. By (10) we can therefore write

$$
\begin{aligned}
\sum_{i=2}^{q} \alpha_{i}^{2} & =\left\|\left[\begin{array}{c}
\sum_{i=2}^{q_{1}} \alpha_{i} u_{i} \\
\sum_{i=q_{1}+1}^{q} \alpha_{i} u_{i}
\end{array}\right]\right\|^{2}=\frac{4}{c_{1}^{2}}\left\|\left[\begin{array}{c}
\sum_{i=2}^{q_{1}} \frac{c_{1}}{2} \alpha_{i} u_{i} \\
\sum_{i=q_{1}+1}^{q} \frac{c_{1}}{2} \alpha_{i} u_{i}
\end{array}\right]\right\|^{2} \leq \frac{4}{c_{1}^{2}}\left\|\left[\begin{array}{c}
\sum_{i=2}^{q_{1}} \alpha_{i}\left(\sigma_{1}-\sigma_{i}+h\right) u_{i} \\
\sum_{i=q_{1}+1}^{q} \alpha_{i}\left(\sigma_{1}-\sigma_{i}+h\right) u_{i}
\end{array}\right]\right\|^{2} \\
& \leq \frac{16 w^{2}\|B\|^{4}}{c_{1}^{2}}
\end{aligned}
$$


This lets us have

$$
\begin{aligned}
\|D u\| & =\left\|D\left[\begin{array}{c}
\alpha_{1} u_{1} \\
0
\end{array}\right]+D\left[\begin{array}{c}
\sum_{i=2}^{q_{1}} \alpha_{i} u_{i} \\
\sum_{i=q_{1}+1}^{q} \alpha_{i} u_{i}
\end{array}\right]\right\| \\
& \geq\left|\alpha_{1}\right| \cdot\left\|D\left[\begin{array}{c}
u_{1} \\
0
\end{array}\right]\right\|-\|D\| \cdot\left\|\left[\begin{array}{c}
\sum_{i=2}^{q_{1}} \alpha_{i} u_{i} \\
\sum_{i=q_{1}+1}^{q} \alpha_{i} u_{i}
\end{array}\right]\right\| \\
& \geq c_{2}\left(1-\frac{16 w^{2}\|B\|^{4}}{c_{1}^{2}}\right)^{1 / 2}-\|D\| \frac{4 w\|B\|^{2}}{c_{1}} .
\end{aligned}
$$

Combining (91) and (11) yields $\|D u\|>0$, meaning $u \notin$ null D. Subcase 2.2: $\sigma_{1}=0$. When $\sigma_{1}=0$ we have $R u=h u$. That $R$ is symmetric positive semidefinite implies that the eigenvalue $h$ is nonnegative. Now, $h$ cannot be zero because then we have $R u=0$ which implies $u \in \operatorname{span}\left\{\mathbf{1}_{q}\right\}$, contradicting our initial assumption. Hence $h>0$. Note that $\sigma_{1}=0$ yields $u_{1}=\mathbf{1}_{q_{1}} / \sqrt{q_{1}}$ since the laplacian $R_{1}$ represents a connected component. Now, the second block of $R^{-}$has also a an eigenvalue at the origin which is not repeated because $R_{2}$ too represents a connected component. Without loss of generality we can let this eigenvalue be $\sigma_{q}=0$. This means $u_{q}=\mathbf{1}_{q_{2}} / \sqrt{q_{2}}$. Observing the orthogonal relationships $\mathbf{1}_{q}^{T} u=0$, $\mathbf{1}_{q_{1}}^{T} u_{i}=0$ for $i=2,3, \ldots, q_{1}$, and $\mathbf{1}_{q_{2}}^{T} u_{i}=0$ for $i=q_{1}+1, q_{1}+2, \ldots, q-1$; we obtain the identity $q_{2} \alpha_{q}^{2}=q_{1} \alpha_{1}^{2}$ through

$$
\begin{aligned}
\alpha_{1} \sqrt{q_{1}}+\alpha_{q} \sqrt{q_{2}} & =\left(\mathbf{1}_{q_{1}}^{T} u_{1}\right) \alpha_{1}+\sum_{i=2}^{q_{1}}\left(\mathbf{1}_{q_{1}}^{T} u_{i}\right) \alpha_{i}+\left(\mathbf{1}_{q_{2}}^{T} u_{q}\right) \alpha_{q}+\sum_{i=q_{1}+1}^{q-1}\left(\mathbf{1}_{q_{2}}^{T} u_{i}\right) \alpha_{i} \\
& =\mathbf{1}_{q_{1}}^{T} \sum_{i=1}^{q_{1}} \alpha_{i} u_{i}+\mathbf{1}_{q_{2}}^{T} \sum_{i=q_{1}+1}^{q} \alpha_{i} u_{i}=\mathbf{1}_{q}^{T} u=0 .
\end{aligned}
$$

Therefore

$$
\left(1+q_{1} / q_{2}\right) \alpha_{1}^{2}=\alpha_{1}^{2}+\alpha_{q}^{2}=1-\sum_{i=2}^{q-1} \alpha_{i}^{2}
$$

Now, by (10) we can write

$$
\begin{aligned}
\sum_{i=2}^{q-1} \alpha_{i}^{2} & =\left\|\left[\begin{array}{c}
\sum_{i=2}^{q_{1}} \alpha_{i} u_{i} \\
\sum_{i=q_{1}+1}^{q-1} \alpha_{i} u_{i}
\end{array}\right]\right\|^{2}=\frac{4}{c_{1}^{2}}\left\|\left[\begin{array}{c}
\sum_{i=2}^{q_{1}} \frac{c_{1}}{2} \alpha_{i} u_{i} \\
\sum_{i=q_{1}+1}^{q-1} \frac{c_{1}}{2} \alpha_{i} u_{i}
\end{array}\right]\right\|^{2} \leq \frac{4}{c_{1}^{2}}\left\|\left[\begin{array}{c}
\sum_{i=2}^{q_{1}} \alpha_{i}\left(\sigma_{1}-\sigma_{i}+h\right) u_{i} \\
\sum_{i=q_{1}+1}^{q} \alpha_{i}\left(\sigma_{1}-\sigma_{i}+h\right) u_{i}
\end{array}\right]\right\|^{2} \\
& \leq \frac{16 w^{2}\|B\|^{4}}{c_{1}^{2}} .
\end{aligned}
$$

This, (12), and the identity $\alpha_{q}=-\alpha_{1} \sqrt{q_{1} / q_{2}}$ allow us write

$$
\begin{aligned}
\|D u\| & =\left\|D\left[\begin{array}{l}
\alpha_{1} u_{1} \\
\alpha_{q} u_{q}
\end{array}\right]+D\left[\begin{array}{c}
\sum_{i=2}^{q_{1}} \alpha_{i} u_{i} \\
\sum_{i=q_{1}+1}^{q-1} \alpha_{i} u_{i}
\end{array}\right]\right\| \\
& \geq\left\|\alpha_{1} D\left[\begin{array}{c}
\frac{\mathbf{1}_{q_{1}}}{\sqrt{q_{1}}} \\
-\frac{\sqrt{q_{1}}}{\sqrt{q_{2}}} \frac{\mathbf{1}_{q_{2}}}{\sqrt{q_{2}}}
\end{array}\right]\right\|-\|D\| \cdot\left\|\left[\begin{array}{c}
\sum_{i=2}^{q_{1}} \alpha_{i} u_{i} \\
\sum_{i=q_{1}+1}^{q-1} \alpha_{i} u_{i}
\end{array}\right]\right\| \\
& \geq\left|\alpha_{1}\right| c_{2} \sqrt{1+q_{1} / q_{2}}-\|D\|\left(\sum_{i=2}^{q-1} \alpha_{i}^{2}\right)^{1 / 2} \\
& \geq c_{2}\left(1-\frac{16 w^{2}\|B\|^{4}}{c_{1}^{2}}\right)^{1 / 2}-\|D\| \frac{4 w\|B\|^{2}}{c_{1}} .
\end{aligned}
$$

Combining (9) and (13) yields $\|D u\|>0$, meaning $u \notin$ null $D$. Hence, in neither of the subcases (when $\sigma_{1}>0$ and when $\left.\sigma_{1}=0\right)$ the laplacian $R$ can have an eigenvector in the set null $D \backslash \operatorname{span}\left\{\mathbf{1}_{q}\right\}$. Then we deduce by Lemma 3 that the interconnection $\left(\mathcal{V}, \mathcal{B}_{\mathrm{d}}, \mathcal{B}_{\mathrm{r}}\right)$ has structural synchronization property. 


\section{Strong structural synchronization}

It directly follows from the definitions that strong structural synchronization implies structural synchronization. We later present examples where an SS network fails to be SSS. That is to say, SS and SSS are not equivalent. This brings up the question: Under what conditions does an SS interconnection become SSS? The below theorem presents one such characterization.

Theorem 2 Let the triple $\left(\mathcal{V}, \mathcal{B}_{\mathrm{d}}, \mathcal{B}_{\mathrm{r}}\right)$ has structural synchronization property. And let $G_{\mathrm{d}}$ and $G_{\mathrm{r}}$ be the incidence matrices of the graphs $\left(\mathcal{V}, \mathcal{B}_{\mathrm{d}}\right)$ and $\left(\mathcal{V}, \mathcal{B}_{\mathrm{r}}\right)$, respectively. Then the interconnection $\left(\mathcal{V}, \mathcal{B}_{\mathrm{d}}, \mathcal{B}_{\mathrm{r}}\right)$ has strong structural synchronization property if and only if either $\mathcal{B}_{\mathrm{r}}=\emptyset$ or else the equation $\operatorname{sgn}\left(G_{\mathrm{r}}^{T} G_{\mathrm{r}} x\right)=\operatorname{sgn}(x)$ has no solution $x$ in the set null $G_{\mathrm{d}}^{T} G_{\mathrm{r}} \backslash\{0\}$.

Proof. Let us be given an SS interconnection $\mathcal{G}=\left(\mathcal{V}, \mathcal{B}_{\mathrm{d}}, \mathcal{B}_{\mathrm{r}}\right)$. We consider two possibilities separately. Case 1: $\mathcal{B}_{\mathrm{r}}=\emptyset$. Since $\mathcal{G}$ is SS, by Theorem 1 the graph $\left(\mathcal{V}, \mathcal{B}_{\mathrm{d}} \cup \mathcal{B}_{\mathrm{r}}\right)$ is connected. Therefore the graph $\left(\mathcal{V}, \mathcal{B}_{\mathrm{d}}\right)$ is connected because $\mathcal{B}_{\mathrm{r}}$ is empty. Thence null $G_{\mathrm{d}}^{T}=\operatorname{span}\left\{\mathbf{1}_{q}\right\}$. Let $D \in \operatorname{lap}\left(\mathcal{V}, \mathcal{B}_{\mathrm{d}}\right)$ be an arbitrary laplacian. Since null $D=$ null $G_{\mathrm{d}}^{T}$ we have null $D=\operatorname{span}\left\{\mathbf{1}_{q}\right\}$. This yields null $D \backslash \operatorname{span}\left\{\mathbf{1}_{q}\right\}=\emptyset$. Then by Lemma 3 we trivially have $\operatorname{Re} \lambda_{2}(D+j R)>0$ for all $R \in \operatorname{lap}\left(\mathcal{V}, \mathcal{B}_{\mathrm{r}}\right)$. That is, $\mathcal{G}$ is SSS.

Case 2: $\mathcal{B}_{\mathrm{r}} \neq \emptyset$. Suppose $\mathcal{G}$ is not SSS. Then there exist two diagonal matrices $\Lambda_{\mathrm{d}}$ and $\Lambda_{\mathrm{r}}$, both with positive diagonal entries, such that $\operatorname{Re} \lambda_{2}\left(D_{1}+j R_{1}\right) \leq 0$ for $D_{1}=G_{\mathrm{d}} \Lambda_{\mathrm{d}} G_{\mathrm{d}}^{T}$ and $R_{1}=G_{\mathrm{r}} \Lambda_{\mathrm{r}} G_{\mathrm{r}}^{T}$. This implies, by Lemma 3 , that there exists an eigenvector $u \notin \operatorname{span}\left\{\mathbf{1}_{q}\right\}$ of $R_{1}$ satisfying $D_{1} u=0$. Let $\lambda$ be the corresponding eigenvalue, i.e., $R_{1} u=\lambda u$. Note first that $\lambda \geq 0$ because $R_{1}$ is symmetric positive semidefinite. Also, $\lambda \neq 0$; for, otherwise, (i.e., if $\lambda=0$ ) we would simultaneously have $R_{1} u=0$ and $D_{1} u=0$, which would imply $u \in \operatorname{null} G_{\mathrm{r}}^{T} \cap \operatorname{null} G_{\mathrm{d}}^{T}$. But since $\mathcal{G}$ is SS, the graph $\left(\mathcal{V}, \mathcal{B}_{\mathrm{d}} \cup \mathcal{B}_{\mathrm{r}}\right)$ is connected by Theorem 1, meaning null $G_{\mathrm{r}}^{T} \cap$ null $G_{\mathrm{d}}^{T}=\operatorname{span}\left\{\mathbf{1}_{q}\right\}$. This would imply that $u$ belongs to the set $\operatorname{span}\left\{\mathbf{1}_{q}\right\}$, which it doesn't. Therefore $\lambda>0$. Define $y=\Lambda_{\mathrm{r}} G_{\mathrm{r}}^{T} u$. The vector $y$ is nonzero because if $y=0$ then we would have the following contradiction $0=G_{\mathrm{r}} y=G_{\mathrm{r}} \Lambda_{\mathrm{r}} G_{\mathrm{r}}^{T} u=R_{1} u=\lambda u \neq 0$. We can write

$$
\begin{aligned}
\lambda y & =\Lambda_{\mathrm{r}} G_{\mathrm{r}}^{T}(\lambda u) \\
& =\Lambda_{\mathrm{r}} G_{\mathrm{r}}^{T} R_{1} u \\
& =\Lambda_{\mathrm{r}} G_{\mathrm{r}}^{T} G_{\mathrm{r}} \Lambda_{\mathrm{r}} G_{\mathrm{r}}^{T} u \\
& =\Lambda_{\mathrm{r}} G_{\mathrm{r}}^{T} G_{\mathrm{r}} y .
\end{aligned}
$$

Since $\lambda>0$ and $\Lambda_{\mathrm{r}}$ is a diagonal matrix with positive diagonal entries, (14) implies $\operatorname{sgn}(y)=\operatorname{sgn}\left(G_{\mathrm{r}}^{T} G_{\mathrm{r}} y\right)$. Furthermore, since $D_{1} u=0$ implies $G_{\mathrm{d}}^{T} u=0$, we have $y \in$ null $G_{\mathrm{d}}^{T} G_{\mathrm{r}}$ as can be seen from

$$
G_{\mathrm{d}}^{T} G_{\mathrm{r}} y=G_{\mathrm{d}}^{T} G_{\mathrm{r}} \Lambda_{\mathrm{r}} G_{\mathrm{r}}^{T} u=G_{\mathrm{d}}^{T} R_{1} u=\lambda G_{\mathrm{d}}^{T} u=0 .
$$

To show the other direction, suppose now that there exists a nonzero vector $x$ simultaneously satisfying $\operatorname{sgn}(x)=\operatorname{sgn}\left(G_{\mathrm{r}}^{T} G_{\mathrm{r}} x\right)$ and $G_{\mathrm{d}}^{T} G_{\mathrm{r}} x=0$. Let $D_{2} \in \operatorname{lap}\left(\mathcal{V}, \mathcal{B}_{\mathrm{d}}\right)$ be an arbitrary laplacian. Define $v=G_{\mathrm{r}} x$. First note that $v \notin \operatorname{span}\left\{\mathbf{1}_{q}\right\}$; for, otherwise, we would have $G_{\mathrm{r}}^{T} v=0$ (since $G_{\mathrm{r}}^{T} \mathbf{1}_{q}=0$ ) and the following contradiction would emerge

$$
0=\operatorname{sgn}\left(G_{\mathrm{r}}^{T} v\right)=\operatorname{sgn}\left(G_{\mathrm{r}}^{T} G_{\mathrm{r}} x\right)=\operatorname{sgn}(x) \neq 0
$$

because $x \neq 0$. Also, $G_{\mathrm{d}}^{T} v=G_{\mathrm{d}}^{T} G_{\mathrm{r}} x=0$, yielding $D_{2} v=0$. Hence, we can write $v \in$ null $D_{2} \backslash \operatorname{span}\left\{\mathbf{1}_{q}\right\}$. Let now $\Lambda$ be a diagonal matrix with positive diagonal entries satisfying $x=\Lambda G_{\mathrm{r}}^{T} G_{\mathrm{r}} x$. Such $\Lambda$ exists since $\operatorname{sgn}(x)=\operatorname{sgn}\left(G_{\mathrm{r}}^{T} G_{\mathrm{r}} x\right)$. Define $R_{2}=G_{\mathrm{r}} \Lambda G_{\mathrm{r}}^{T}$. Note that $R_{2} \in \operatorname{lap}\left(\mathcal{V}, \mathcal{B}_{\mathrm{r}}\right)$. Also, $v$ is an eigenvector of $R_{2}$ because we can write $R_{2} v=G_{\mathrm{r}} \Lambda G_{\mathrm{r}}^{T} G_{\mathrm{r}} x=G_{\mathrm{r}} x=v$. Then by Lemma 3 we have $\operatorname{Re} \lambda_{2}\left(D_{2}+j R_{2}\right) \leq 0$. Consequently, $\mathcal{G}$ is not SSS.

\section{$5 \quad$ Examples of SSS networks}

We begin this section by examining some example topologies from the point of view of strong structural synchronization. Then we establish certain generalizations valid for special classes of interconnections. 
Henceforth, for simplicity, we consider triples $\left(\mathcal{V}, \mathcal{B}_{\mathrm{d}}, \mathcal{B}_{\mathrm{r}}\right)$ with disjoint edge sets only, i.e., we let $\mathcal{B}_{\mathrm{d}} \cap \mathcal{B}_{\mathrm{r}}=$ $\emptyset$. We emphasize that here generality is not compromised in exchange for simplicity because strong structural synchronization property of an arbitrary interconnection can always be studied through one with disjoint edge sets. More precisely:

Proposition 2 The triple $\left(\mathcal{V}, \mathcal{B}_{\mathrm{d}}, \mathcal{B}_{\mathrm{r}}\right)$ is $S S S$ if and only if $\left(\mathcal{V}, \mathcal{B}_{\mathrm{d}}, \mathcal{B}_{\mathrm{r}} \backslash \mathcal{B}_{\mathrm{d}}\right)$ is.

Proof. Given $\mathcal{G}=\left(\mathcal{V}, \mathcal{B}_{\mathrm{d}}, \mathcal{B}_{\mathrm{r}}\right)$ define $\overline{\mathcal{G}}=\left(\mathcal{V}, \mathcal{B}_{\mathrm{d}}, \overline{\mathcal{B}}_{\mathrm{r}}\right)$ with $\overline{\mathcal{B}}_{\mathrm{r}}=\mathcal{B}_{\mathrm{r}} \backslash \mathcal{B}_{\mathrm{d}}$. Note that $\mathcal{G}$ is SS when (and only when) $\overline{\mathcal{G}}$ is SS. This follows from Theorem 1 and the fact that $\mathcal{B}_{\mathrm{d}} \cup \mathcal{B}_{\mathrm{r}}=\mathcal{B}_{\mathrm{d}} \cup \overline{\mathcal{B}}_{\mathrm{r}}$. Since structural synchronization is a necessary condition for strong structural synchronization, we shall focus on the case where the interconnections $\mathcal{G}$ and $\overline{\mathcal{G}}$ are SS. Let $G_{\mathrm{d}}, G_{\mathrm{r}}$, and $\bar{G}_{\mathrm{r}}$ be the incidence matrices of the graphs $\left(\mathcal{V}, \mathcal{B}_{\mathrm{d}}\right),\left(\mathcal{V}, \mathcal{B}_{\mathrm{r}}\right)$, and $\left(\mathcal{V}, \overline{\mathcal{B}}_{\mathrm{r}}\right)$, respectively. Since $\overline{\mathcal{B}}_{\mathrm{r}} \subset \mathcal{B}_{\mathrm{r}}$ and $\mathcal{B}_{\mathrm{r}} \backslash \overline{\mathcal{B}}_{\mathrm{r}} \subset \mathcal{B}_{\mathrm{d}}$ we can find matrices $A$, $B$ and write $G_{\mathrm{r}}=\left[\begin{array}{ll}\bar{G}_{\mathrm{r}} & B\end{array}\right]$ and $G_{\mathrm{d}}=\left[\begin{array}{ll}A & B\end{array}\right]$. When $B$ is the empty matrix, the result trivially follows; for then we have $G_{\mathrm{r}}=\bar{G}_{\mathrm{r}}$, yielding $\mathcal{G}=\overline{\mathcal{G}}$. Therefore we henceforth let $B$ have at least one column. We now consider two cases. Case 1: $\overline{\mathcal{B}}_{\mathrm{r}}=\emptyset$. That $\overline{\mathcal{B}}_{\mathrm{r}}$ is empty has two immediate consequences. One of them is that $\overline{\mathcal{G}}$ is SSS by Theorem 2, the other is $G_{\mathrm{r}}=B$. Suppose now that $\mathcal{G}$ is not SSS. Then by Theorem 2 we can

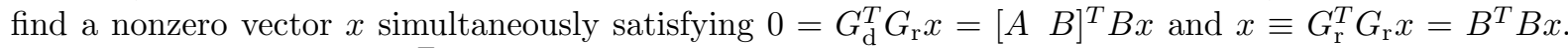
The first equation yields $B^{T} B x=0$. Combining this with the second one implies $x \equiv 0$, resulting in the contradiction $x=0$. Hence $\mathcal{G}$ must be SSS. Case 2: $\overline{\mathcal{B}}_{\mathrm{r}} \neq \emptyset$. Suppose $\mathcal{G}$ fails to be SSS. Then there exists a nonzero vector $x$ satisfying $G_{\mathrm{d}}^{T} G_{\mathrm{r}} x=0$ and $G_{\mathrm{r}}^{T} G_{\mathrm{r}} x \equiv x$. Employing the partitioning $x=\left[x_{1}^{T} x_{2}^{T}\right]^{T}$ we can rewrite these equations as

$$
\begin{aligned}
& {\left[\begin{array}{ll}
A^{T} \bar{G}_{\mathrm{r}} & A^{T} B \\
B^{T} \bar{G}_{\mathrm{r}} & B^{T} B
\end{array}\right]\left[\begin{array}{l}
x_{1} \\
x_{2}
\end{array}\right]=0,} \\
& {\left[\begin{array}{ll}
\bar{G}_{\mathrm{r}}^{T} \bar{G}_{\mathrm{r}} & \bar{G}_{\mathrm{r}}^{T} B \\
B^{T} \bar{G}_{\mathrm{r}} & B^{T} B
\end{array}\right]\left[\begin{array}{l}
x_{1} \\
x_{2}
\end{array}\right] \equiv\left[\begin{array}{l}
x_{1} \\
x_{2}
\end{array}\right] .}
\end{aligned}
$$

Now, (15) yields $B^{T} \bar{G}_{\mathrm{r}} x_{1}+B^{T} B x_{2}=0$ while $B^{T} \bar{G}_{\mathrm{r}} x_{1}+B^{T} B x_{2} \equiv x_{2}$ by (16). Combining these we obtain $x_{2}=0$. Since $x$ was nonzero, we have to have $x_{1} \neq 0$. Moreover, in the light of $x_{2}=0$, the equations (15) and (16) can be reduced to

$$
\begin{aligned}
0 & =\left[\begin{array}{ll}
A & B
\end{array}\right]^{T} \bar{G}_{\mathrm{r}} x_{1}=G_{\mathrm{d}}^{T} \bar{G}_{\mathrm{r}} x_{1}, \\
x_{1} & \equiv \bar{G}_{\mathrm{r}}^{T} \bar{G}_{\mathrm{r}} x_{1} .
\end{aligned}
$$

Thence we deduce by Theorem 2 that $\overline{\mathcal{G}}$ cannot be SSS because $x_{1}$ is nonzero. It is not difficult to see that the steps we have taken can be traced back. That is, SSS of $\bar{G}$ implies SSS for $\mathcal{G}$. The proof is therefore complete.

Example 1. As our first example, let us recall the four-node interconnection (for which we let $\mathcal{V}=$ $\left\{\nu_{1}, \nu_{2}, \nu_{3}, \nu_{4}\right\}$ be the vertex set) we visited earlier in the paper, reproduced in Fig. 2 where the resistor represents the (only) edge in the set $\mathcal{B}_{\mathrm{d}}=\left\{\left\{\nu_{1}, \nu_{3}\right\}\right\}$ and the inductors represent by the edges in $\mathcal{B}_{\mathrm{r}}=$ $\left\{\left\{\nu_{1}, \nu_{2}\right\},\left\{\nu_{2}, \nu_{3}\right\},\left\{\nu_{3}, \nu_{4}\right\}\right\}$. By Theorem 1 this interconnection $\left(\mathcal{V}, \mathcal{B}_{\mathrm{d}}, \mathcal{B}_{\mathrm{r}}\right)$ is clearly SS. To check whether it is also SSS, let us apply the test presented in Theorem 2 , Note that the incidence matrices

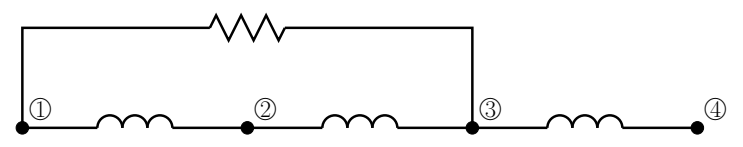

Figure 2: An interconnection that is not SSS. The edges in $\mathcal{B}_{\mathrm{d}}$ are depicted as resistors; the edges in $\mathcal{B}_{\mathrm{r}}$ are depicted as inductors.

associated to $\left(\mathcal{V}, \mathcal{B}_{\mathrm{d}}, \mathcal{B}_{\mathrm{r}}\right)$ read

$$
G_{\mathrm{d}}=\left[\begin{array}{r}
1 \\
0 \\
-1 \\
0
\end{array}\right], \quad G_{\mathrm{r}}=\left[\begin{array}{rrr}
1 & 0 & 0 \\
-1 & 1 & 0 \\
0 & -1 & 1 \\
0 & 0 & -1
\end{array}\right]
$$


Choosing, for instance, the vector $x=\left[\begin{array}{lll}2 & -1 & 1\end{array}\right]^{T}$ yields $G_{\mathrm{r}} x=\left[\begin{array}{llll}2 & -3 & 2 & -1\end{array}\right]^{T}$, whence follows $G_{\mathrm{r}}^{T} G_{\mathrm{r}} x=\left[\begin{array}{lll}5 & -5 & 3\end{array}\right]^{T}$ and, consequently, $G_{\mathrm{r}}^{T} G_{\mathrm{r}} x \equiv x$. Moreover, we have $G_{\mathrm{d}}^{T} G_{\mathrm{r}} x=0$. Therefore the interconnection is not SSS.

A somewhat physical interpretation of the above equations is possible in the following way. Let the $i$ th column of the incidence matrix $G_{\mathrm{r}}$ be $\left[e_{k}-e_{\ell}\right]$. Then if the $i$ th entry of the vector $x$ is $c_{k \ell}$, we can say a current of value $c_{k \ell}$ flows through the edge $\left\{\nu_{k}, \nu_{\ell}\right\} \in \mathcal{B}_{\mathrm{r}}$ from $\nu_{k}$ to $\nu_{\ell}$; or, equivalently, a current of value $-c_{k \ell}$ flows from $\nu_{\ell}$ to $\nu_{k}$. That is, the direction of any current can be reversed by changing the sign of its value. This allows us, without loss of generality, to consider only the case where all currents are nonnegative. As for the edges that belong to $\mathcal{B}_{\mathrm{d}}$, we assign them zero currents, which makes their directions immaterial. Moreover, the mapping $x \mapsto G_{\mathrm{r}} x$ can be interpreted as that the edge currents $(x)$ generate the vertex potentials $\left(G_{\mathrm{r}} x\right)$ through the rule:

(A1) The potential of a vertex equals the sum of outgoing currents minus the sum of incoming currents associated to that vertex.

Remark $1 A$ direct implication of (A1) is that the vertex potentials throughout any interconnection always add up to zero.

Having defined edge currents and vertex potentials now we can interpret the constraints $G_{\mathrm{d}}^{T} G_{\mathrm{r}} x=0$ and $G_{\mathrm{r}}^{T} G_{\mathrm{r}} x \equiv x$ easily. The former means:

(A2) The potentials of any two vertices that are connected through an edge that belongs to $\mathcal{B}_{\mathrm{d}}$ are equal.

Whereas $G_{\mathrm{r}}^{T} G_{\mathrm{r}} x \equiv x$ is equivalent to:

(A3) If two vertices with different potentials are connected by an edge then the current on that edge is positive and flows from higher potential to lower potential. And if two vertices have the same potential then the current through the edge that connects them is zero.

The edge currents $\left(\left[\begin{array}{lll}2 & -1 & 1\end{array}\right]^{T}=x\right)$ and the vertex potentials $\left(\left[\begin{array}{llll}2 & -3 & 2 & -1\end{array}\right]^{T}=G_{\mathrm{r}} x\right)$ we have used in our example are shown in Fig. 3, where we observe that all three conditions (A1), (A2), (A3) are satisfied. Let us now formalize our observations.

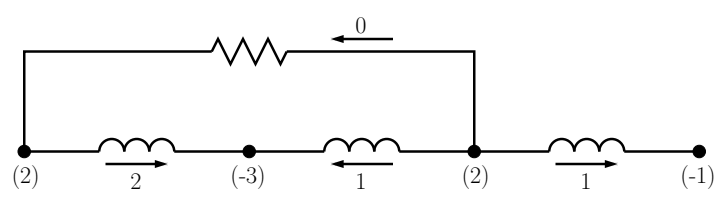

Figure 3: The vertex potentials (shown in brackets) and edge currents of Example 1.

Definition 3 Given an $S S$ interconnection $\left(\mathcal{V}, \mathcal{B}_{\mathrm{d}}, \mathcal{B}_{\mathrm{r}}\right)$ let $\mathcal{D}$ denote some (compatible) distribution where each edge is assigned a (nonnegative) current and each vertex a potential; obeying the rules (A1), (A2), and (A3). The distribution $\mathcal{D}$ is said to be nontrivial if it has at least one nonzero current, otherwise it is called trivial.

This definition lets us make a restatement of Theorem 2.

Theorem 3 An SS interconnection is SSS if and only if it does not admit a nontrivial distribution.

Example 2. This time consider the interconnection shown in Fig. 4h. Let us analyze it using our last theorem. Let $\mathcal{D}$ be an arbitrary distribution, where the potential $p_{1}$ of the vertex (1) is $\alpha$. Then by (A2) we have $p_{4}=p_{1}=\alpha$, whence follows, by Remark 1 and (A2), $p_{2}=p_{3}=-\alpha$. These vertex potentials are shown in Fig. 4 $\mathrm{b}$. Let us now focus on the edge currents $c_{12}, c_{13}, c_{43}$ shown in the same figure. Applying rule (A1) on the second and fourth vertices yields $c_{12}=\alpha$ and $c_{43}=\alpha$, respectively. Finally, we apply it on the first vertex and obtain $c_{12}+c_{13}=\alpha$, whence we deduce $c_{13}=0$. Now, that the current 


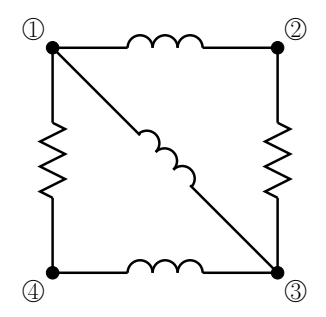

(a)

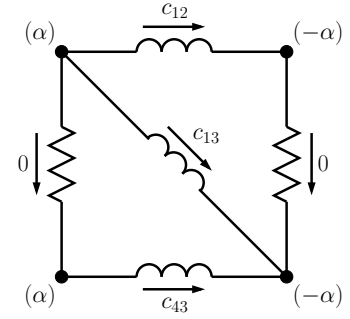

(b)

Figure 4: An SSS interconnection. (The edges in $\mathcal{B}_{\mathrm{d}}$ are depicted as resistors; the edges in $\mathcal{B}_{\mathrm{r}}$ are depicted as inductors.)

$c_{13}$ is zero implies by (A3) that the first and third vertices must have the same potential $p_{1}=p_{3}$, i.e., $\alpha=-\alpha$, yielding $\alpha=0$. This means that the distribution $\mathcal{D}$ is trivial. Theorem 3 then tells us that the interconnection is SSS.

Further examples are given in Fig. 5, where a nontrivial current distribution (from which, by (A1), the potential distribution can be determined) is provided for the instances that are not SSS. In the light of Theorem 3 we next provide certain graph theoretical conditions that guarantee SSS for special types of topologies; namely, path graphs, cycles, and trees. But this requires a quick review of some graph terminology first.

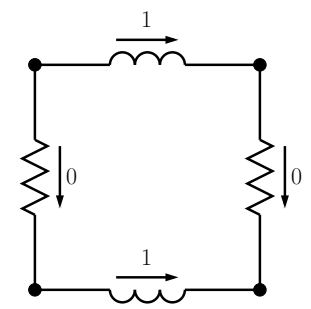

(a)

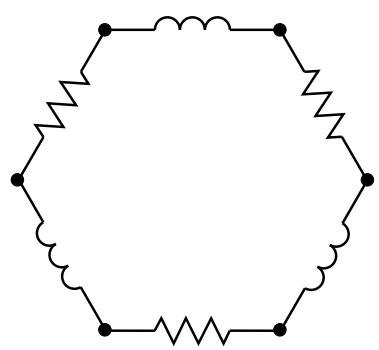

(b)

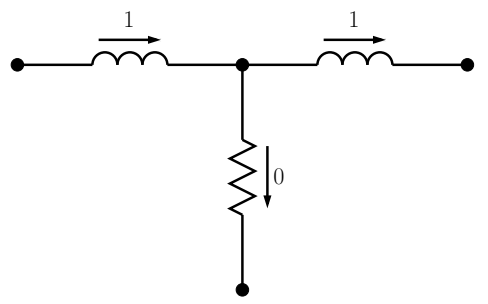

(c)

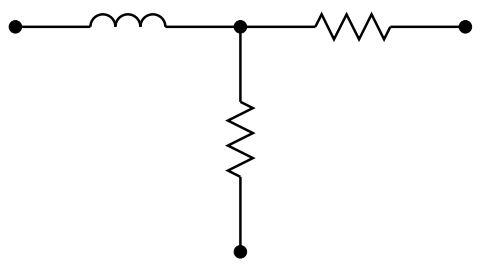

(d)

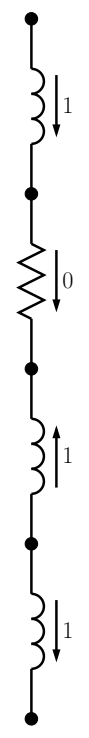

(e)

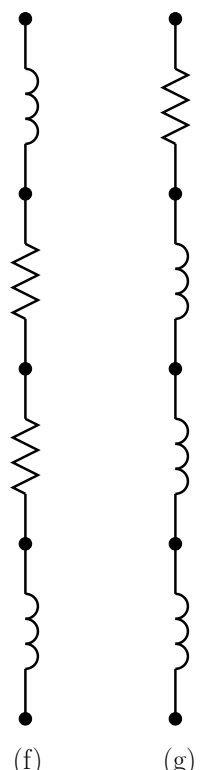

(f) (g)

Figure 5: Various interconnections. The topologies (b), (d), (f), and (g) are SSS; whereas (a), (c), and (e) are not SSS. (The edges in $\mathcal{B}_{\mathrm{d}}$ are depicted as resistors; the edges in $\mathcal{B}_{\mathrm{r}}$ are depicted as inductors.)

Let $\Gamma=(\mathcal{V}, \mathcal{B})$ be a graph with the vertex set $\mathcal{V}=\left\{\nu_{1}, \nu_{2}, \ldots, \nu_{q}\right\}$ and $q \geq 2$. The graph $\Gamma$ is a path if the edge set can be written as $\mathcal{B}=\left\{\left\{\nu_{1}, \nu_{2}\right\},\left\{\nu_{2}, \nu_{3}\right\}, \ldots,\left\{\nu_{q-1}, \nu_{q}\right\}\right\}$ perhaps after a relabeling of the vertices; it is a cycle if $\mathcal{B}=\left\{\left\{\nu_{1}, \nu_{2}\right\},\left\{\nu_{2}, \nu_{3}\right\}, \ldots,\left\{\nu_{q-1}, \nu_{q}\right\},\left\{\nu_{q}, \nu_{1}\right\}\right\}$. Namely, the incidence 
matrices of a path and a cycle have the following structures

$$
G_{\text {path }}=\left[\begin{array}{rrrrr}
1 & 0 & 0 & \cdots & 0 \\
-1 & 1 & 0 & \cdots & 0 \\
0 & -1 & 1 & \cdots & 0 \\
\vdots & \vdots & \vdots & \ddots & \vdots \\
0 & 0 & 0 & \cdots & 1 \\
0 & 0 & 0 & \cdots & -1
\end{array}\right]_{q \times(q-1)} \quad, \quad G_{\text {cycle }}=\left[\begin{array}{rrrrrr}
1 & 0 & 0 & \cdots & 0 & -1 \\
-1 & 1 & 0 & \cdots & 0 & 0 \\
0 & -1 & 1 & \cdots & 0 & 0 \\
\vdots & \vdots & \vdots & \ddots & \vdots & \vdots \\
0 & 0 & 0 & \cdots & 1 & 0 \\
0 & 0 & 0 & \cdots & -1 & 1
\end{array}\right]_{q \times q}
$$

Note that the $i j$ th entry of $G_{\text {path }}$ reads

$$
\left[G_{\mathrm{path}}\right]_{i j}=\left\{\begin{aligned}
-1 & \text { for } i=j+1, \\
1 & \text { for } i=j, \\
0 & \text { elsewhere }
\end{aligned}\right.
$$

A tree is a generalization of path in the sense that its incidence matrix $G_{\text {tree }}$ satisfies (perhaps after a suitable relabeling)

$$
\left[G_{\text {tree }}\right]_{i j}=\left\{\begin{array}{cl}
-1 & \text { for } i=j+1 \\
0 \text { or } 1 & \text { for } i \leq j \\
0 & \text { elsewhere }
\end{array}\right.
$$

in addition to the usual constraint that each column is of the form $\left[e_{k}-e_{\ell}\right]$. A vertex $\nu_{i}$ of a tree is called a leaf if the $i$ th row of the incidence matrix contains only a single nonzero entry. For instance, a path has exactly two leaves: first and the last vertices. Henceforth for a given $\left(\mathcal{V}, \mathcal{B}_{\mathrm{d}}, \mathcal{B}_{\mathrm{r}}\right)$ we denote by $\left[\mathcal{B}_{\mathrm{r}}\right] \subset \mathcal{V}$ the set of vertices that are associated to the edges in $\mathcal{B}_{\mathrm{r}}$. That is, $\left[\mathcal{B}_{\mathrm{r}}\right]=\left\{\nu \in \mathcal{V}: \nu \in\left\{\nu_{i}, \nu_{j}\right\} \in \mathcal{B}_{\mathrm{r}}\right\}$. The set $\left[\mathcal{B}_{\mathrm{d}}\right]$ is defined similarly. We now list some straightforward consequences of Theorem 3 ,

Corollary 1 Let the interconnection $\mathcal{G}=\left(\mathcal{V}, \mathcal{B}_{\mathrm{d}}, \mathcal{B}_{\mathrm{r}}\right)$ be such that $\mathcal{B}_{\mathrm{d}} \cap \mathcal{B}_{\mathrm{r}}=\emptyset$ and the graph $\left(\mathcal{V}, \mathcal{B}_{\mathrm{d}} \cup \mathcal{B}_{\mathrm{r}}\right)$ is a path. Then $\mathcal{G}$ is $S S S$ if and only if $\mathcal{V} \backslash\left[\mathcal{B}_{\mathrm{r}}\right]$ is not empty.

Corollary 2 Let the interconnection $\mathcal{G}=\left(\mathcal{V}, \mathcal{B}_{\mathrm{d}}, \mathcal{B}_{\mathrm{r}}\right)$ be such that $\mathcal{B}_{\mathrm{d}} \cap \mathcal{B}_{\mathrm{r}}=\emptyset$ and the graph $\left(\mathcal{V}, \mathcal{B}_{\mathrm{d}} \cup \mathcal{B}_{\mathrm{r}}\right)$ is a cycle with $q$ nodes. Then $\mathcal{G}$ is SSS if and only if

1. Either $\mathcal{V} \backslash\left[\mathcal{B}_{\mathrm{r}}\right]$ is not empty,

2. Or else $\mathcal{V} \backslash\left[\mathcal{B}_{\mathrm{d}}\right]$ is empty and $q / 2$ is odd.

Corollary 3 Let the interconnection $\mathcal{G}=\left(\mathcal{V}, \mathcal{B}_{\mathrm{d}}, \mathcal{B}_{\mathrm{r}}\right)$ be such that $\mathcal{B}_{\mathrm{d}} \cap \mathcal{B}_{\mathrm{r}}=\emptyset$ and the graph $\Gamma=$ $\left(\mathcal{V}, \mathcal{B}_{\mathrm{d}} \cup \mathcal{B}_{\mathrm{r}}\right)$ is a tree. Then $\mathcal{G}$ is $S S S$ if $\left[\mathcal{B}_{\mathrm{r}}\right]$ contains at most one leaf of $\Gamma$.

Let us revisit the interconnections in Fig. [ 5 in the light of these corollaries. The interconnections (e), (f), and (g) are paths for which the set $\mathcal{V} \backslash\left[\mathcal{B}_{\mathrm{r}}\right]$ is empty only for (e). By Corollary 1 therefore the path (e) is not SSS, while the paths (f) and (g) are. The interconnections (a) and (b) are cycles, and clearly $\mathcal{V} \backslash\left[\mathcal{B}_{\mathrm{r}}\right]$ is empty for both. In this case Corollary 2 advises us to check $\mathcal{V} \backslash\left[\mathcal{B}_{\mathrm{d}}\right]$, which too is empty for both. It is not difficult to see that when $\mathcal{V} \backslash\left[\mathcal{B}_{\mathrm{r}}\right]$ and $\mathcal{V} \backslash\left[\mathcal{B}_{\mathrm{d}}\right]$ are simultaneously empty for a cycle it must be that the sets $\mathcal{B}_{\mathrm{r}}$ and $\mathcal{B}_{\mathrm{d}}$ must contain equal number of edges. This means that the number of nodes $q$ is necessarily an even number, yielding that $q / 2$ is an integer. For (a) we have $q / 2=2$, an even number, while for (b) $q / 2=3$ is odd. Via Corollary 2 we arrive therefore at the conclusion that only the cycle (b) is SSS. Finally, consider the trees (c) and (d). The tree (c) has two leaves that belong to $\left[\mathcal{B}_{\mathrm{r}}\right]$. In such a case we cannot use Corollary 3 since the condition given there is only sufficient. For the other tree, however, the set $\left[\mathcal{B}_{\mathrm{r}}\right]$ contains a single leaf. Hence the interconnection (d) must be SSS.

\section{Conclusion}

In this paper we presented a structural analysis of synchronization in linear networks of identical oscillators (e.g. LC circuits) coupled through both dissipative connectors (e.g. resistors) and restorative connectors 
(e.g. inductors). We provided answers to the following two questions. First, for a given coupling structure, when can we find a suitable set of coupling strengths that guarantees asymptotic synchronization of the oscillators? Second, for what type of structures is synchronization guaranteed regardless of the coupling strengths? The answer to the first question turned out to be very simple: A suitable choice of parameter values (yielding synchronization) exists when the network is connected and is not entirely free of dissipative coupling. The second problem however admitted only a more elaborate solution, which required us to introduce flow diagrams (defined through three relatively nontechnical rules) for our analysis. This solution yielded simple conditions on synchronization for networks whose coupling topology is either a path or a cycle or a tree.

\section{References}

[1] S. Celikovsky, V. Lynnyk, and G. Chen. Robust structural synchronization in dynamical complex netwroks. In Proc. of the 7th IFAC Symposium on Nonlinear Control Systems, pages 264-269, 2007.

[2] G.H. Golub and C.F. Van Loan. Matrix Computations (Third Edition). The Johns Hopkins University Press, 1996.

[3] B. Y. Hou, X. Li, and G. Chen. Structural controllability of temporally switching networks. IEEE Transactions on Circuits and Systems I, 63:1771-1781, 2016.

[4] C.-T. Lin. Structural controllability. IEEE Transactions on Automatic Control, 19:201-208, 1974.

[5] Y.-Y. Liu and A.-L. Barabasi. Control principles of complex systems. Reviews of Modern Physics, 88:035006, 2016.

[6] H. Mayeda and T. Yamada. Strong structural controllability. SIAM Journal on Control and Optimization, 17:123-138, 1979.

[7] G. Parlangeli and G. Notarstefano. On the reachability and observability of path and cycle graphs. IEEE Transactions on Automatic Control, 57:743-748, 2012.

[8] A. Rahmani, M. Ji, M. Mesbahi, and M. Egerstedt. Controllability of multi-agent systems from a graph-theoretic perspective. SIAM Journal on Control and Optimization, 48:162-186, 2009.

[9] W. Ren. Synchronization of coupled harmonic oscillators with local interaction. Automatica, 44:3195$3200,2008$.

[10] S.E. Tuna. Synchronization of linear oscillators coupled through a dynamic network with interior nodes, 2019. arXiv:1906.08572 [math.DS].

[11] X. L. Wang, H. S. Su, L. Wang, and X. F. Wang. Edge consensus on complex networks: a structural analysis. International Journal of Control, 90:1584-1596, 2017.

[12] L. Xiang, F. Chen, W. Ren, and G. Chen. Advances in network controllability. IEEE Circuits and Systems Magazine, 2:8-32, 2019.

[13] X. Xin. Linear strong structural controllability and observability of an $n$-link underactuated revolute planar robot with active intermediate joint or joints. Automatica, 94:436-442, 2018.

[14] S. Zhang, M. Cao, and M. K. Camlibel. Upper and lower bounds for controllable subspaces of networks of diffusively coupled agents. IEEE Transactions on Automatic Control, 59:745-750, 2014. 